\title{
Stress Recovery for the Particle-in-cell Finite Element Method
}

Authors: Haibin Yang ${ }^{1,2}$, Louis N. Moresi ${ }^{1,2}$, John Mansour ${ }^{3}$

Affiliation 1: School of Earth Sciences, University of Melbourne, Melbourne, Australia

Affiliation 2: Research School of Earth Sciences, Australian National University, Canberra, Australia

Affiliation 3: Monash eResearch Centre, Monash University, Clayton, Australia

Corresponding Author: Haibin Yang

Email:haibiny@student.unimelb.edu.au

This manuscript has been submitted for publication in Physics of the Earth and Planetary Interiors. Please note that, despite having undergone peer-review, the manuscript has yet to be formally accepted for publication. Subsequent versions of this manuscript may have slightly different content. If accepted, the final version of this manuscript will be available via the 'Peer-reviewed Publication DOI' link on the right-hand side of this webpage. Please feel free to contact any of the authors; we welcome feedback. 


\section{Abstract}

The particle-in-cell Finite Element (PIC-FE) method has been widely used in geodynamic numerical modelling due to its efficiency in dealing with large deformations without the requirement of remeshing. However, material deformation within a Eulerian mesh frame will mix particles of contrasting strength properties (e.g., viscosity in Stokes problems) in a single element requiring some form of averaging to project particle properties to integration points. The numerical solutions are thus dependent on the way how the particle properties are projected to the integration points. An intra-element property discontinuity may introduce severe stress oscillations along the interfaces. In this study, we assess three preprocessing methods to smooth the viscosity contrast within one element. For simplified models with analytical solutions, the accuracy and convergence rate in $L_{2}$ norm are systematically studied with ensembles. It is found that using higher-order quadrature elements does not improve the convergence rate for either the velocity or stress solution, both close to one. Additionally, the convergence rate of the maximum stress error, which exists adjacent to the mixed-material elements, is much less than one for all cases studied here. Comparing each component of the stress tensor, we find that the stress tensor component with the highest strain rate gradient across the material interface produces the maximum stress error. Such errors can be reduced by averaging the particle properties to the Gaussian quadrature point with an inverse-distance-weighted harmonic mean.

\section{Key words:}

Particle-in-cell; Finite Element Method; Stress fluctuation; Stress smoothing; Numerical geodynamic modelling 


\section{Introduction}

The classical finite element method (FEM) (see e.g. Hughes (2012)) has been widely used to simulate diverse problems in engineering. Unlike most engineering problems, geological simulations are dominated by emergence of geometrical structures due to the non-linear processes involved (Lenardic et al., 2003). For the structurally conforming FEM meshes, the large deformations result in distorted meshes, which often require repeated remeshing in computation (Braun and Sambridge, 1994; Davies et al., 2011; Maddison and Hiester, 2017). The particle-incell (PIC) method allows Lagrangian material particles to move in a background Eulerian mesh (Harlow, 1964; Poliakov and Podladchikov, 1992; Sulsky et al., 1994; Moresi et al., 2002; Thielmann and Kaus, 2012; Puckett et al., 2018). Those particles carry the density, composition, viscosity, etc., while the unknowns are solved at nodes of the mesh. In addition to the particle-in-cell FEM, the PIC finite difference (FD) method, where the governing equation is discretized using the finite difference method, is also widely used in geodynamic numerical models (Gerya and Yuen, 2003; Deubelbeiss and Kaus, 2008; Duretz et al., 2011).

PIC implementations have many advantages for problems where large-deformation and emergent structures are dominant, but there are two shortcomings that appear if the mesh does not conform to interfaces that separate materials of different strength: the first is that the interface is represented by a continuous interpolant on the mesh which can introduce significant errors in calculating fluxes such as the stress-tensor; the second problem relates to the discretization of the boundary shape by a non-conforming mesh which introduces corners and "staircases" into the geometry that are not present in the original interface. This can produce severe stress- 
focusing artefacts.

In the tectonic-modelling problem, interfaces typically represent boundaries between materials of different viscosity (effective viscosity in the case of a visco-elastic problem) or the location of a shear band with distinctive constitutive properties from the intact material. A viscosity jump within one element may give rise to errors more than two orders of magnitude larger than where material interfaces align with element boundaries (Moresi et al., 1996). This viscosity jump can also significantly degrade the convergence properties of numerical solvers (May and Moresi, 2008; May, 2009; Thielmann et al., 2014; Gassmöller et al., 2019). For modelling long-term geological problems, the stress artifacts could dissipate this error to historical evolution with non-linear processes (Bardenhagen and Kober, 2004).

To address the effect of mixing materials of contrasting viscosity in one element, one strategy is to divide the element into sub-domains along the interfaces between different materials within one cell, and integrate those with same properties separately (Braun et al., 2008; Wallstedt and Guilkey, 2011). As subdivision of the mesh by searching for material interfaces increases the computational complexity (Puckett et al., 2018), smoothing the discontinuities by averaging particle properties with the arithmetic or harmonic mean (Deubelbeiss and Kaus, 2008; Schmeling et al., 2008) or using a smooth basis function in the FEM (Bardenhagen and Kober, 2004) can be a simpler approach. Averaging of the viscosity in cells with multi-phase materials in the FD method was systematically studied by Deubelbeiss and Kaus (2008). However, they did not make full use of the possibilities offered by the FEM, as they use a single value for all quadrature points in one element. This constant interpolation of viscosity in the cell with mixed materials is also tested by Thielmann et al. (2014), who proposed another more sophisticated strategy, the linear least square interpolation, which requires solving a linear equation for each mixed-material 
element. However, this extra cost of combining linear least square interpolation with $\mathrm{Q}_{2}$ elements does not work well in cases of sharp changes of viscosity in one element (Thielmann et al., 2014).

We examine the effectiveness of several smoothing methods in eliminating spurious stress fluctuations within the framework of Underworld, which is a Python package used to simulate geodynamics processes (Moresi et al., 2007; Mansour et al., 2020). The smoothing methods we test can be conveniently implemented and efficiently run with Underworld. We first introduce two post-processing methods available in Underworld that are used to compute a smooth nodal stress or strain rate field, and then describe three pre-processing solutions that can be used to mitigate errors associated with the mesh not conforming to the interfaces: (1) a node-based method using the element shape functions, (2) an element-based averaging method, and (3) a Gauss-quadrature-point-based method using the distance weighted averaging. Additionally, we further compare them with the classical stress recovery technique that reconstructs continuous stresses on specified patches based on nearby super-convergent points (SPR) (Zienkiewicz and Zhu, 1992a, b). The SPR method was designed for cases without the internal structure in the element and is thus not intended to alleviate the problem caused by mixed-material elements. Each method is tested against analytic solutions for models that are relevant to geological problems. We also discuss how best to combine the various pre- and post-processing methods in "real" modelling situations. To be comparable with previous studies (May, 2009; $\underline{\text { Duretz et al., }}$ 2011; Thielmann et al., 2014), the accuracy and convergence rate in the $L_{2}$ norm for the global model scale are provided. Additionally, we discuss the local maximum error, which is not addressed in previous studies but is of importance for geological problems such as shear band development where there is a non-linear feedback that can be sensitive to mesh-dependent errors. 


\section{Governing equations}

The simulation is based on the Stokes equation for Newtonian viscous, incompressible flow:

$$
\begin{gathered}
\frac{\partial \sigma_{i j}}{\partial x_{j}}-\frac{\partial P}{\partial x_{i}}=\rho g_{i} \\
\frac{\partial v_{i}}{\partial x_{i}}=0 \\
\sigma_{i j}=2 \eta \dot{\varepsilon}_{i j} \\
\dot{\varepsilon}_{i j}=\frac{1}{2}\left(\frac{\partial v_{i}}{\partial x_{j}}+\frac{\partial v_{j}}{\partial x_{i}}\right)
\end{gathered}
$$

where $\sigma_{i j}$ denotes deviatoric stress, $P$ pressure, $\rho$ density, $g_{i}$ gravity acceleration, $v_{i}$ velocity, $\eta$ Newtonian viscosity, $\dot{\varepsilon}_{i j}$ strain rate, and the Einstein summation convention is used here.

\section{Methods}

\subsection{Post-processing methods}

In the classical Finite Element Method, inter-element stress continuity is not guaranteed. Smoothing the numerical discontinuity to fit the physically continuous system has been conventionally implemented as a post-processing step (Zienkiewicz and Zhu, 1992a, b; Boroomand and Zienkiewicz, 1997b). These generally include (1) averaging around local nodes and (2) global projection with least square fits (Hinton and Campbell, 1974).

\subsubsection{Averaging around local nodes with shape functions}

The projection at mesh nodes is done through extrapolation from superconvergent points (e.g., 
Gaussian points) and then averaging locally at mesh nodes. In the FEM framework, the distancebased weight can be found using the shape function $\left(N_{a}\right)$ in the whole calculation domain $\Omega$, so the average nodal stress is

$$
\sigma^{h}=\frac{\int N_{a} \sigma^{p} d \Omega}{\int N_{a} d \Omega}
$$

where $\sigma^{p}$ is the point stress computed in the element interior. Note that, although the integration is applied to the whole domain, the shape function has compact support, limited to adjacent elements. In this study, we adopt the convention that the superscript $p$ and $h$ denote the properties from points in the element interiors and mesh nodes, respectively.

\subsubsection{Global projection with least square fits}

The least square fit approach can be applied to the whole finite element domain to minimize the error between the smoothed stresses $\tilde{\sigma}$ and the point stresses $\sigma^{p}$

$$
\int\left(\tilde{\sigma}-\sigma^{p}\right)^{2} d \Omega
$$

The smoothed stress is written as

$$
\tilde{\sigma}=\sum_{B=1}^{n_{n p}} N_{B} \sigma_{B}^{h}
$$

where $n_{n p}$ is the number of nodal points, $N_{B}$ the respective shape function and $\sigma_{B}^{h}$ the stress on the mesh node $B$. The least squares method is used to minimize the error by

$$
\frac{\partial}{\partial \sigma_{B}^{h}} \int\left(\tilde{\sigma}-\sigma^{p}\right)^{2} d \Omega=0
$$

for $B=1,2, \ldots, n_{n p}$. This yields the following matrix problem: 


$$
\boldsymbol{X} \widetilde{\boldsymbol{\sigma}}=\boldsymbol{P}
$$

where $\boldsymbol{X}=\left[X_{\boldsymbol{A} \boldsymbol{B}}\right], \widetilde{\boldsymbol{\sigma}}=\left\langle\sigma_{B}^{h}>\right.$ and $\boldsymbol{P}=\left\langle P_{A}>\right.$.

The construction of $\boldsymbol{X}$ and $\boldsymbol{P}$ is implemented in elementwise fashion:

$$
x_{a b}^{e}=\int_{\Omega_{e}} N_{a}^{e} N_{b}^{e} d \Omega, \quad p_{a}^{e}=\int_{\Omega_{e}} \sigma_{p} N_{a}^{e} d \Omega
$$

for $1 \leq a, b \leq n_{e n}$, where $n_{e n}$ is the number of nodal points per element.

It has to be noted that the global projection method is a more costly process to recover accurate nodal stresses and may also produce overshoot values at nodal points (Thielmann et al., 2014).

\subsubsection{SPR-superconvergent point recovery}

The SPR method (Zienkiewicz and Zhu, 1992a) computes a continuous nodal stress field, $\sigma^{*}$, from a patch of elements local to each node:

$$
\sigma^{*}=M a
$$

where $\boldsymbol{M}=\left[\mathbf{1}, x, x^{2}, \ldots, x^{m}\right]$ and $\boldsymbol{a}=\left[a_{1}, a_{2}, a_{3}, \ldots, a_{m+1}\right]^{\boldsymbol{T}}$ for the one-dimensional cases. $m$ is the order of the polynomial expansion, which is the same as that used in the shape function $N$. Accordingly, for the two-dimensional expansion for linear elements, $\boldsymbol{M}=[1, x, y]$, and for quadratic $\boldsymbol{M}=\left[1, x, y, x^{2}, x y, y^{2}\right]$ (the $x y$ term is optional).

To determine the unknown parameters $\boldsymbol{a}$ in equation (11), we minimize

$$
\boldsymbol{F}=\sum_{i=1}^{n}\left(\sigma_{i}^{h}\left(x_{i}, y_{i}\right)-\boldsymbol{M}\left(x_{i}, y_{i}\right) \boldsymbol{a}\right)^{2}
$$

where $\left(x_{i}, y_{i}\right)$ are the coordinates of specified sampling points, the total number of which is $n$. Therefore, for $\boldsymbol{F}$ to be a minimum 


$$
\frac{\partial \boldsymbol{F}}{\partial \boldsymbol{a}_{\boldsymbol{i}}}=0
$$

This gives

$$
\sum_{i=1}^{n} \boldsymbol{M}^{\mathbf{T}}\left(x_{i}, y_{i}\right) \boldsymbol{M}\left(x_{i}, y_{i}\right) \boldsymbol{a}=\sum_{i=1}^{n} \boldsymbol{M}^{\mathbf{T}}\left(x_{i}, y_{i}\right) \sigma^{h}\left(x_{i}, y_{i}\right)
$$

This system is rewritten as

$$
a=A^{-1} b
$$

where

$$
\boldsymbol{A}=\sum_{i=1}^{n} \boldsymbol{M}^{\mathbf{T}}\left(x_{i}, y_{i}\right) \boldsymbol{M}\left(x_{i}, y_{i}\right) \text { and } \boldsymbol{b}=\sum_{i=1}^{n} \boldsymbol{M}^{\mathbf{T}}\left(x_{i}, y_{i}\right) \sigma^{h}\left(x_{i}, y_{i}\right)
$$

After obtaining the parameter $\boldsymbol{a}$ in equation (11), with the polynomial expansion, the mesh node values can be evaluated at any given coordinates using the functional form of $\sigma^{*}$ in equation

(11). Although clearly an expensive method, SPR is a node-by-node technique that only needs to be computed when and where accurate stresses are required. We note that momentum conservation is not guaranteed in the SPR method, but a more sophisticated approach can reconstruct the stress by equilibrium of patches using original momentum equations (Boroomand and Zienkiewicz, 1997b, a; May, 2009).

\subsection{Pre-processing methods}

The classical post-processing methods are designed to produce continuous, node-based stress field, but not to resolve the stress perturbations caused by mixed-material elements. Instead, we utilize pre-processing methods to reduce the strength contrast across the interfaces between two materials. Directly sampling the nearest particle to Gaussian points is taken as the reference method, which is named as Direct in the following sections. 
To smooth the strength properties in one cell, the arithmetic mean (Am), geometric mean $(\mathrm{Gm})$ and harmonic mean $(\mathrm{Hm})$ are commonly used. For a positive viscosity sequence $\boldsymbol{\eta}=$ $\eta_{1}, \eta_{2}, \ldots, \eta_{n}$, where $n$ is the total number of components in the sequence, they are defined respectively by

$$
\operatorname{Am}(\boldsymbol{\eta})=\frac{1}{n} \sum_{i=1}^{n} \eta_{i} \operatorname{Gm}(\boldsymbol{\eta})=\sqrt[n]{\prod_{i=1}^{n} \eta_{i}} \text { and } \operatorname{Hm}(\boldsymbol{\eta})=n / \sum_{i=1}^{n} 1 / \eta_{i}
$$

It is well known and has been demonstrated by Xia et al. (1999) that for the same sequence,

Am $\geq \mathrm{Gm} \geq \mathrm{Hm}$. The harmonic mean produces an averaged value close to the minimum of the sequence while the arithmetic mean produces a result close to the maximum of the sequence. In terms of the physical meaning, Schmeling et al. (2008) suggested that the harmonic-mean averaging best represents the effective viscosity of simple shear models, while the arithmeticmean averaging can stand for the effective viscosity of pure shear models.

For the FD method, the harmonic-mean averaging has been found to be the optimal smoothing method in the viscosity field (Deubelbeiss and Kaus, 2008). However, for a buoyancy driven system, the arithmetic averaging for density is the dominant factor rather than the viscosity (Deubelbeiss and Kaus, 2008). For the FEM, the results from Thielmann et al. (2014) also indicated that the harmonic mean averaging method is the preferred one to smooth the viscosity. Therefore, we mainly test two end-member averaging methods: the harmonic and arithmetic averaging method for the viscosity field in this study.

\subsubsection{Node-based method}

We use the method described in section 3.1.1 to project reciprocals of the property information $\eta^{p}$ (e.g., viscosity) carried by particles to mesh nodes through the shape function 
$\left(N_{a}\right)$ in the whole calculation domain $\Omega$ (Fig. 1a) :

$$
\eta^{h}=\frac{\int N_{a}\left(1 / \eta^{p}\right) d \Omega}{\int N_{a} d \Omega}
$$

The strength values $\eta^{*}$ considered in the elementwise integration is

$$
\eta^{*}=\sum_{A=1}^{n_{n p}} N_{A}\left(1 / \eta_{A}^{h}\right)
$$

\subsubsection{Element-based method}

Here we first look for elements that contain more than one material, and then unify the properties of all the particles in those elements to be one value (Fig. 1b) (Deubelbeiss and Kaus, 2008; Thielmann et al., 2014). The harmonic mean method averaging over all types of points in one cell gives the unified property value

$$
\eta^{e}=n_{k} / \sum_{A=1}^{n_{k}} 1 / \eta_{A}^{p}
$$

where $n_{k}$ is the number of material types in one element. It is worthwhile noting that $n_{k}$ is not the number of particles in one element and it is different from what other codes do (Deubelbeiss and Kaus, 2008; Thielmann et al., 2014). For large viscosity jump $>10^{3} \mathrm{~Pa} \cdot \mathrm{s}$, if the particles numbers in one cell is few (e.g., 10s), the effective viscosity for both cases are at the same order of magnitude. For simplicity, we take the number of material types.

\subsubsection{Gaussian-quadrature-point based method}

This method is also applied only to elements with a mixture of materials, and we first locate mixed-material cells. Then, instead of unifying materials over one cell, particles within distance $\delta$ 
to the selected Gauss quadrature point (Fig. 1C) are averaged by

$$
\eta^{g p}=\sum_{A=1}^{n_{d p}} W_{A}^{p} / \sum_{A=1}^{n_{d p}} W_{A}^{p} / \eta_{A}^{p}
$$

where $n_{d p}$ is the number of sampling points for the specified Gauss quadrature point and $W_{A}^{p}$ is the weight. The sampling numbers $n_{d p}$ is determined by the selected distance $\delta$. The effect of sampling distance is discussed later. The distance weighted averaging is found to produce good results in the FD method (Duretz et al., 2011), therefore the weight used in this study depends on the reciprocal of the distance from the sampled particle to the Gauss quadrature point. The default averaging method is harmonic mean as shown in equation (21), and the arithmetic averaging method (eq. 17) is also fully investigated here. The Gaussian-quadraturepoint based method with harmonic mean averaging over a distance of $\delta$ is recorded as Gauss$\mathrm{Hm} \delta$ for short, while the one with the arithmetic mean strategy is Gauss -Am $\delta$. In this study, $\delta=$ $0.5,1,2,4$ times of the corresponding element size at different resolutions.

\subsection{Error measurement}

To study the error caused by intra-element viscosity jumps, we use a set of models with existing analytical solutions. To solve the governing equations, we only use direct solvers (e.g., LU or MUMPS with large positive penalty values) rather than the iterative solvers for numerical experiments. The later need subjective criteria to stop iterative cycles, while the direct solver only introduces errors on the order of floating-point precision on the computer. We compute the error by the normalized root mean square error in $L_{2}$ norm 


$$
\text { RMS error }=\sqrt{\frac{\int\left(\varphi_{\text {ana }}-\varphi_{\text {num }}\right)^{2} d \Omega}{\int \varphi_{\text {ana }}{ }^{2} d \Omega}}
$$

where $\varphi_{\text {ana }}$ and $\varphi_{\text {num }}$ are the analytical and numerical values, respectively; The integration is over the domain $\Omega$, which is an elementwise calculation in the FEM. Additionally, we also measure the maximum error

$$
\text { Maximum error }=\max \left(\left|\varphi_{\text {ana }}-\varphi_{\text {num }}\right|\right)
$$

which is a pointwise measurement over the whole calculation domain. The convergence rate $r$ is measured by computing the numerical solution under a sequence of regular meshes with the $L_{2}$ norm error determined in equation (22)

\section{RMS error $\leq C h^{r}$}

where $h$ is the mesh size and $C$ is a $h$-independent constant. With the commonly used $\mathrm{Q}_{k} \times$ $\mathrm{Q}_{k-1}($ subscript $\mathrm{k}$ is the element degree) 'Taylor-Hood' elements (Taylor and Hood, 1973) for the velocity and pressure discretization, the optimal convergence rate $r$ for the error of the velocity and pressure in the $L_{2}$ norm is $k+1$ and $k$, respectively (Bercovier and Pironneau, 1979). In this study, we test cases with $\mathrm{k}=1$ and 2, which are named as $\mathrm{Q}_{1}$ and $\mathrm{Q}_{2}$ elements. The orders of accuracy of the RMS and Maximum errors between analytical and numerical solutions in velocity and shear stress for different smoothing methods are listed in Table A1. The details of the convergence rate study are discussed in the next section. 


\section{Results and discussion}

\subsection{Models with analytical solutions}

\subsubsection{Simple shear model with regular mesh}

We first test the effect of different smoothing methods on a simple shear model (Fig. 2a). The dimensionless model ranges from -1 to 1 in both $x$ and $y$ directions and has two layers with a

viscosity contrast $\frac{\eta_{1}}{\eta_{2}}=10^{3}$. The driving velocity $V_{0}$ is 1 and periodic boundary conditions are applied to the left and right boundaries. Rectangular meshes of $N \times N$ are compared, where $N$ $=15,33,75,133$ for $\mathrm{Q}_{1}$ elements, and $N=7,17,37,67$ for $\mathrm{Q}_{2}$ elements. Taking the solution of Couette flow and considering stress continuity at the interface, the analytical solution for the shear stress reads as

$$
\sigma_{x y}=\frac{2 V_{0} \eta_{2}}{\frac{h_{1} \eta_{2}}{\eta_{1}}+h_{2}}
$$

where $h_{1}$ and $h_{2}$ are the thickness of two layers. The coordinate of the material interface $\left(y_{\text {interface }}\right)$ is designed around $y=0$ with $\left|y_{\text {interface }}\right|<d y / 2$, where $d y$ is one element size in $y$ direction for the corresponding mesh resolution. For the mixed-material cells around $y=0$, if the interface is at a height of $\alpha$ of the element size, $h_{1}=1-(\alpha-0.5) \times d y$ and $h_{2}=1+(\alpha-0.5) \times d y$. We create model ensembles with $\alpha=0.1,0.15,0.25,0.35,0.5,0.75,0.9 . \alpha=0.5$ represents the case with the interface located exactly in the middle of the box $(y=0)$.

The detailed convergence study for each $V_{x}$ and $\sigma_{x y}$ of the ensembles is shown in Fig. 3a 
and 3b, where we directly sampling the nearest particle property to the Gauss quadrature point and project the stress to local nodes with the shape functions (Direct0 in Fig. $3 \mathrm{c}$ and $3 \mathrm{~d}$ ). The order of convergence in velocity is one, which, however, is supposed to be second order in theory without the effect of material mixing in one element (May, 2009). The stress convergence rate is less than one. The RMS error for both the velocity and stress increases by one order of magnitude with $\alpha$ increasing from 0.1 to 0.9 . That means how two materials are mixed in one cell has significant influences on the both the velocity and stress accuracy evaluation. If we test the accuracy and convergence rate by varying model resolution with a fixed interface, and do not have such ensemble test, models with different resolutions may correspond to different $\alpha$ values in the ensembles. This would cause fluctuations in the RMS error, thus biasing our assessment of the convergence rate with linear regression analysis. With the ensembles, the convergence rate is calculated with the linear regression for the mean value of the ensembles at corresponding mesh size (Fig. $3 \mathrm{c}$ and $3 \mathrm{~d}$ ). The ranges of the upper and lower limit in the ensembles are shown with vertical bars with caps in Fig. $3 c$ and $3 d$. This rule applies to all the convergence rate calculation for models with analytical solutions in this study.

Changing the number of particles in one element almost has no effect on the results for the Direct0 case (Fig. 3c and 3d). As we use the nearest sampling method to project particle properties to Gaussian point, only those closest to the respective Gaussian point contribute to the projection. In this case, increasing particle numbers much higher than the number of Gaussian points will not change solutions too much for the models which have a relatively simple geometry. The particle per cell numbers should also have little effect on the element-based method, as it unifies the element properties by the numbers of material types rather than the particle numbers. For 
smooth problems that do not have intra-element viscosity discontinuities, it has been suggested that increasing particle per cell numbers with mesh resolution may increase the convergence rate (Gassmöller et al., 2019). However, increasing the number of particles comes at the cost of an increased computation time. In the following sections, we fix the number of particles in each element to be 32 for both $Q_{1}$ and $Q_{2}$ elements, where $Q_{1}$ and $Q_{2}$ elements have $3 \times 3$ and $5 \times 5$ Gauss quadrature points, respectively. The selected particle per cell number is higher than the respective number of Gaussian points and we find the case with 32 particles works as accurately as the case with 128 particles per cell (not shown in Figures). Therefore, the number of 32 can be a good compromise between accuracy and computational cost. Additionally, the simple case shows that the global projection of least square fits (Direct1 in Fig. 3c and 3d) produces worse results than the local projection with shape functions (Direct0 in Fig. $3 \mathrm{c}$ and $3 \mathrm{~d}$ ). We only use the local projection method to obtain the stress on mesh nodes in the following sections and focus more on the effect of different pre-processing methods.

The calculated stress field with the mesh resolution of 133 for $\mathrm{Q}_{1}$ elements with $\alpha=0.5$ is shown in Fig. 2. The maximum stress error along the interface reaches $66 \%$ of the analytical solution for the node-based method (Fig. 2c). The element-based method produces the best result in this particularly simple case with a maximum error of $2 \%$ (Fig. $2 \mathrm{~d}$ ). Increasing the sampling radius in the Gauss-Hm method helps to lower the maximum error (Fig. 2e-h). Further increasing the sampling distance should produce similar results as the element-based method, but it would increase computational time as well. The optimal sampling distance is thus the crucial parameter in the Gaussian-quadrature-point based method. 
For the ensembles calculated with $\mathrm{Q}_{1}$ elements, the convergence rate for the RMS error is $\sim 1$ in velocity and $<1$ in stress for the cases processed with all the methods studied here (Fig. 4). The element-based method produces the lowest RMS error in stress, but the highest RMS error in velocity (Fig. 4a and 4b). The Gauss-Hm4 method produces a mean RMS error in stress as low as the element-based method, but the RMS error in velocity is also as high as that with the element-based method. In terms of the RMS error, the optimal distance is two-element widths for the Gauss-Hm method, which produces a slightly higher mean RMS error in velocity, but a much lower mean RMS error in stress than that in the one-element-width case (Fig. 4a and 4b). For the arithmetic mean averaging method (Gauss-Am), it neither reduces the velocity error with respect to the Direct method, nor produces better results than the harmonic mean method in terms of the RMS error of stress (Fig. $4 \mathrm{c}$ and $4 \mathrm{~d}$ ).

In the cases of $\mathrm{Q}_{2}$ elements (Fig. 5), the convergence rate for the RMS error in velocity is still approximately close to one for $\mathrm{Q}_{1}$ elements and does not increase as the classical FEM theory predicts. That means using higher-order quadrature integration may not improve the convergence rate when there are mixing materials with contrasting viscosity in one element, as has also been suggested by May 2009. The node-based method better recovers the velocity field than other methods tested in this study but produces the worst result in stress. The Gaussianquadrature-point based method with the harmonic mean averaging over a sampling radius of one element size (Gauss-Hm1) is the preferred method as it results in the lowest RMS error in stress and second lowest error in velocity. Comparing with the Direct method, we find the GaussAm method almost does not damp the RMS error in velocity, nor does it result in a better stress recovery than the harmonic mean method. 


\subsubsection{Simple shear model with distorted mesh}

The numerical experiments illustrated in section 4.1.1 are a specific case study where meshes are aligned with the material interface. In this study, with all else being equal, we perturb mesh around $y=0$. For meshes nodes with $|y|<2 d y$, where $d y$ is one element size in $y$ direction for the corresponding mesh resolution as well, the mesh nodes are deformed following $y=y_{0}+$ $\beta \times d y \times \sin (2 \pi k x)$ with $k$ being the wave number, which is set to be 4 in this study, $\beta$ the factor that affects the amplitude of the perturbation and $y_{0}$ the original $y$ coordinates in the regular mesh. The interface in the study is fixed at $y=0$, and we test the model ensembles with $\beta=0.1,0.3,0.6,0.8$.

The case of $\beta=0.8$ with the resolution of $133 \times 133$ for $\mathrm{Q}_{1}$ elements is shown in Fig. 6. As the element-based method unifies material properties in elements that contain particles of different properties, it produces sharp contact boundaries horizontally (Fig. 6a). Such sharp contact is conventionally known to introduce a "staircase" effect, which is suggested to reduce the velocity error convergence rate $\left(L_{2}\right.$ norm $)$ from $O\left(h^{2}\right)$ to $O\left(h^{1}\right)$ for $Q_{1}$ rectangular elements in the classical FEM theory (Ramière, 2008). The "staircase" effect is unable to be explored in section 4.1.1, which indicated that the element-based method has advantages in reducing stress perturbations. With a more general case like that shown in Fig. 6a, the element-based method produces poor stress field results (Fig. 6b) with the maximum error of $52 \%$ relative to the analytical solution., The Gauss-Hm2 method reduces its maximum error to $41 \%$ of the analytical solution

(Fig. 6c). Further applying the SPR postprocessing method to the stress field obtained by Gauss- 
Hm2 lowers the maximum error down to 27 \% (Fig. 6d).

The convergence rate study for the ensembles of different $\beta$ is shown in Fig. 7-8. For $\mathrm{Q}_{1}$ elements (Fig. 7), the pattern RMS error does not differ much from that in section 4.1.1. The convergence rate for both velocity and stress approximate one. Comparing with the $\mathrm{Q}_{1}$ element in section 4.1 .1 (Fig. 4), we find that the irregular mesh has increased the mean RMS error stress by a factor of 5-10 for the element-based method and the Gauss-Hm2 method, which might have enhanced the "staircase" effect due to the distorted mesh (Fig. 6). The optimal sampling distance for the Gauss-Hm method is two element sizes, which equals the value found in section 4.1.1. It is interesting to note that the node-based method generates the narrowest range and lowest mean value of RMS errors both in velocity and stress field.

Comparing the RMS error of $\mathrm{Q}_{2}$ elements in Fig. 8 with those in Fig. 5 in section 4.1.1, we find the node-based method displays major changes with the widest range and the highest value for both the RMS error. On the other hand, the node-based method also leads to the highest convergence rate among all tested methods. The convergence rate in both velocity and stress for the node-based method is more than twice of that with other methods. This might be caused by the high-order shape functions used to project the particle properties to the interpolation points, which was also found to improve the convergence rate for smooth problems (Gassmöller et al., 2019). 


\subsubsection{SolCx model}

The SolCx benchmark model was used by Thielmann et al. (2014) to test the influence of a sharp viscosity jump within one element on computation error and convergence rate. The SolCx benchmark is a complementary case to the previous model which includes the body force terms which are not considered in the previous simple shear models. The analytic solution for this case was derived by Zhong (1996), and the code for this analytic solution is included in the Underworld package.

The model consists of a unit box where density is described by the trigonometric function $\rho=\sin (\pi y) \cos (\pi x)$. Gravity is 0 in the $x$ direction and 1 in the $y$ direction (Fig. 9). Free-slip boundary conditions are applied to all boundaries. As before, rectangular meshes of $N \times N$ are compared, where $N=15,33,75,133$ for $\mathrm{Q}_{1}$ elements, and $N=7,17,37,67$ for $\mathrm{Q}_{2}$ elements. There is a viscosity jump of $10^{3}$ at $x=0.5+\gamma d x$, where $d x$ is one element size in $\mathrm{x}$ direction for the corresponding mesh resolution, and $\gamma$ is a constant as $\alpha$ in section 4.1.1. We build model ensembles with $\gamma=0.1,0.15,0.25,0.35,0.5,0.75,0.9$.

If $\mathrm{Q}_{1}$ elements are used (Fig. 10), RMS errors display the same behaviour as observed in the simple shear models. The convergence rate of the RMS error in velocity and stress is about one. The major change we observe in this case is that the Gauss-Hm method does not reduce the mean RMS error in stress with respect to the Direct method as efficiently as in section 4.1 .1 and 4.1.2, but still lowers the upper limit of the RMS error in stress by a factor of 2 for the Gauss - Hm2 method. Further applying the SPR method, it reduces the stress RMS error by $10 \%$ with respect 
to the Gauss-Hm2 method (Fig. 10b). The Gauss-Am method recovers a poor velocity field and does not recover the stress field better than the Gauss-Hm method (Fig. 10cd), and the behavior is consistent as in section 4.1.1 and 4.1.2.

The RMS error in $\mathrm{Q}_{2}$ elements (Fig. 11) demonstrates the same pattern as before, and the Gauss-Hm1 method results in the lowest error. The node-based method produces the widest range and highest value of for the RMS error in stress. The convergence rate for velocity and stress is about one for the RMS error for all methods studied here. The higher convergence rate for cases with the node-based method in $\mathrm{Q}_{2}$ elements than other methods observed in section 4.1.2 is not observed in this case.

We note that all the stress convergence rate studies beforehand only consider $\sigma_{x y}$ with $\sigma_{x}$ and $\sigma_{y}$ not being considered. We thus further investigate the RMS error for the $\sigma_{x}, \sigma_{y}$ and pressure, which are calculated with $\mathrm{Q}_{1}$ elements (Fig. 12). It is found that almost all the methods produce the same results, except the node-based method which produces higher error in pressure than others. No significant difference is observed between the Gauss-Hm and GaussAm method. We find that the largest stress errors are located at the interface in the vicinity of mixed-material elements (Fig. 13 a-c). The error shown in Fig. 13a-c is normalized by its maximum value of each component. The error in $\sigma_{x y}$ is in the range of $0-30 \%$ while the error in the other two components is in the range of $0-8 \%$. Additionally, the error in $\sigma_{x y}$ is mainly distributed in the central part while the errors for the other two components are close to the top and base boundaries. With the strain rate distribution for each component (Fig. 13d-f), we find that the maximum errors in each component are all located in regions with large strain rate 
gradients. The higher in strain rate gradient across the interface, the higher error in stress. The gradient of $\dot{\varepsilon}_{x y}$ across the interface is much higher than the two normal components. Such higher strain rate of $\dot{\varepsilon}_{x y}$ across the interface is also the common feature in the simple shear cases in section 4.1.1 and 4.1.2.

We therefore suggest that the harmonic mean averaging method for cases where there are high gradients of tangential strain rate across the material interface. This is consistent with the suggestion by Schmeling et al. (2008) that harmonic-mean averaging best represents the effective viscosity of simple shear models and arithmetic-mean averaging best represents that of pure shear models. For a buoyancy driven system as the SolCx model or other complex geological problems, it may be a composite of both simple shear and pure shear models. Thus, the choice of arithmetic or harmonic mean depends on the specific geologic problem. For the SolCx model, the Gauss-Hm method almost produces comparable, though not better, results for two normal stress components as the Gauss-Am method (Fig. 13). Note that further applying the SPR postprocessing method on those results obtained by the Gauss-Hm may improve the final accuracy (Fig. 10). Therefore, the Gauss-Hm method plus the SPR post-processing method could be a practical option for models when we are not familiar with the dominating deformation mode. Alternatively, it is worthwhile to consider the transversely isotropic viscosity that takes different averaging method for the viscosity in different directions in future work (Sharples et al., 2016).

\subsubsection{Maximum Error}

The convergence rate for the RMS errors reflects how the global error decays with mesh sizes but 
cannot reflect how the local errors vary with the resolution. The maximum error, which exists in mixed-material elements, is thus studied in this section. The results for the three simple models with analytical solutions are found to have common features in terms of the maximum error. The results for the simple shear model with regular mesh calculated with $\mathrm{Q}_{1}$ are shown as an example here, and other models can be found in the appendix.

Regarding the maximum error for $\mathrm{Q}_{1}$ elements (Fig. 14), the convergence rate for velocity is close to 1 , but the maximum error in stress almost does not decay while increasing the resolution (Fig. 14a and 14b). The element-based method is poor in recovering the velocity field as it does in the RMS error, though it produces the lowest stress error among all methods studied here. The Gauss-Am method behaves in the same way as it in the RMS error, neither reducing the maximum error in velocity with respect to the Direct sampling method, nor producing better results than the Gauss-Hm method (Fig. 14c and 14d). The maximum error needs to be treated carefully with the application of yielding stress, which may be lower than the local high stress due to the intraelement strength discontinuities. The spuriously high stress along a shear band may produce a new branch of shear bands, thus affecting the whole geological model evolution. As the maximum error hardly decreases with the mesh resolution for all the methods studied here, more accurate methods are required for further studies.

\subsection{Models withoutanalytical solutions}

Models in sections 4.1 are all based on a relatively simple geometry, the stress field of which can be obtained through analytical solutions. In this section, we test complex models that do not have 
simple analytical solutions. First, we test a model with a fault (thin weak zone) that is at a low angle to the $x$-axis. Then, we build a synthetic model by using the observations from a complex fault network to test how the methods proposed in this study work in a complex system.

\subsubsection{Shear zone at a low angle to $x$-axis}

This model (Fig. 15) has a weak zone $\left(10^{19} \mathrm{~Pa} \cdot \mathrm{s}\right)$ embedded in a strong matrix $\left(10^{23} \mathrm{~Pa} \cdot \mathrm{s}\right)$. The driving velocity at the top and base is $\pm 0.5 \mathrm{~cm} \mathrm{yr}^{-1}$, respectively. The velocity at both lateral boundaries linearly increases from $-0.5 \mathrm{~cm} \mathrm{yr}^{-1}$ at base $(y=-10 \mathrm{~km})$ to $0.5 \mathrm{~cm} \mathrm{yr}^{-1}$ at top $(\mathrm{y}=10$ $\mathrm{km})$. The weak zone ( $260 \mathrm{~m}$ thick) is at $\sim 10^{\circ}$ to the $x$-axis, thus forming rectangular elements that have both strong and weak materials (Fig. 15a). As a reference, we also build a model where mesh edges are aligned with material interfaces (Fig. 15b). In addition, the mesh in the $y$ direction surrounding the weak zone is refined, with the grid size in the y direction varying from $0.21 \mathrm{~m}$ to $330 \mathrm{~m}$. We assume here that the reference model solution is better at representing the true solution than the non-body-conforming cases and the relative difference between non-bodyconforming cases and the reference model then approximates the error.

Compared with the stress field in the reference model (Fig. 15c), the Direct method introduces intensive perturbations along the fault (Fig. 16a), while other methods demonstrate stress shadows adjacent to the weak zone without visible fluctuations as in the Direct method. In addition to the absolute value of the stress field (left panel in Fig. 16a-d), we also calculate the relative differences between the reference model and the case that contains mixed-material elements (right panel in Fig. 16e-h). We find errors distributed along the strike of the embedded weak zone in the model processed with the Direct method. The element-based method and the 
Gauss-Hm2 method (Fig. 16c, 16d, 16g and 16h) produce the best results with localized errors around the fault tips. The results of the node-based method (Fig. 16b and 16f) are in-between. Note that the stress perturbations for three preprocessed cases are mainly close to the tip, but those in the Direct case have some high stress lobes within the supposed stress shadow area (Fig. 16a and 16e). If those stress lobes within the shadow area was higher than the yielding stress of the material, the model would develop a new branch of faulting zone intersecting the stem of the weak zone rather than through the tips. Such artificial pattern may mislead our understanding of shear bands development in the long-term run. The errors in other cases which focus the error in fault tips may affect the quantitative near-fault stress analysis but does not influence first-order evolution pattern of a fault zone.

\subsubsection{Complex fault geometries}

The case study of a complex fault geometry is based on the San Andreas Fault system in California. which strikes through the state of California and is a transform boundary between the Pacific and North American plates. Many studies have suggested that the San Andreas Fault at different segments accommodates $20-75 \%$ relative motion between these two plates ( $\sim 50 \mathrm{~mm}$ $\left.\mathrm{yr}^{-1}\right)$ (Atwater and Stock, 1998; DeMets and Dixon, 1999; Meade and Hager, 2005). We map the major faults based on the WGCEP (2007 Working group on California Earth-quake Probabilities) fault traces, where fault traces with long-term strain rate of orders of magnitude higher than that of less deformed areas are selected (Bird, 2009) (Fig. 17). We only extract the major trace of the San Andreas fault (SAF) and the Garlock fault (GF) for this case study. The 2-D model is $1110 \mathrm{~km}$ long and $484 \mathrm{~km}$ wide with $400 \times 200 \mathrm{Q}_{1}$ elements. A simple shear boundary condition is applied 
to the base $(y=0 \mathrm{~km})$ with $V_{x}=4 \mathrm{~cm} \mathrm{yr}^{-1}$ and the top $(y=484 \mathrm{~km})$ with $\mathrm{V}_{\mathrm{x}}=0 \mathrm{~cm} \mathrm{yr}^{-1}$, and the velocities at two lateral boundaries linearly decrease from $4 \mathrm{~cm} \mathrm{yr}^{-1}$ (base) to $0 \mathrm{~cm} \mathrm{yr}^{-1}$ (top). The fault zone is set to be $\sim 10 \mathrm{~km}$ thick, and spans $3-5$ grid points, and the viscosity for non-fault area is $10^{23} \mathrm{~Pa} \cdot \mathrm{s}$. The mapped fault area is set to be a constant value of $10^{19} \mathrm{~Pa} \cdot \mathrm{s}$, which corresponds to the strength of the wet quartzite deformed at a temperature of $\sim 500 .{ }^{\circ} \mathrm{C}$ with a strain rate of $10^{-13} \mathrm{~s}^{-1}$ (Ranalli, 1995).

We generate a reference model with a resolution of $1600 \times 800 \mathrm{Q}_{1}$ elements, which is four times higher than the test model in each direction. In the model with a resolution of $400 \times 200$ Q1 elements (Fig. 17a-d), the Direct sampling method produces obvious stress lobes along the Garlock fault, which is not seen in the cases that have been preprocessed with a smooth viscosity field (Fig. 17b-d). The difference with respect to the refence model is taken as the approximate error as in section 4.2.1. The absolute errors (Fig. 17f-i) demonstrate the same effect as in section 4.2.1 that the element-based method and Gauss-Hm2 method generates the best results. The absolute errors of magnitude $>10^{7}$ in the Gauss-Hm2 and element-based method are significantly reduced with respect to the Direct method (Fig. 17 k-n). We note that the width of the fault zone, which has the lowest stress (dark blue color), is widened in the case with the nodebased method (Fig. 17b). The harmonic mean averaging method used in the node-based method might have increased the effective thickness of the weak zone, which was also observed in the study of Schmeling et al. (2008). However, this effect is not obvious in the Gauss-Hm method. The node-based method could smear the low-viscosity particles to neighboring elements through the shape function interpolation, but the Gauss-point-based method limits the lowviscosity effect introduced by the harmonic mean method to the mixed-material elements. 
In addition, we build a model with a resolution of $800 \times 400 \mathrm{Q}_{1}$ elements, which has twice resolution of the Direct case in both directions and is named Direct2 in Fig. 17e. We find the Gauss-Hm2 or element-based method produce comparable results as the Direct2 case (Fig. 17jo), and the absolute error in the Mojave block is even lower than the Direct2 case (Fig. 17j). This is promising in saving the computational time for $3 \mathrm{D}$ models with the Gauss-Hm method or element-based method which can recover the stress field with a relatively low-resolution mesh. Additionally, as demonstrated in the analytical models in section 4.1, we need to be careful with the element-based method that tends to produce a worse velocity field than the Gaussianquadrature-point based method with a relatively short sampling distance.

\section{Conclusions}

We compare the effects of both post-processing and pre-processing methods in dampening the stress perturbations which may be introduced by contrasting viscosity within a single element for the particle-in-cell finite element method. The classical post-processing methods alone cannot eliminate stress oscillations. Instead, using the pre-processing methods to reduce the interface strength contrast first, projecting stress from points to local nodes with shape functions can generate a relatively accurate stress field.

(1) The node-based method introduces the least computational cost and recovers the velocity field best among the three methods. However, it cannot recover the stress field as efficiently as other two methods, especially for models interpolated with $\mathrm{Q}_{2}$ element.

(2) The element-based method has advantages in reducing stress perturbations, but it 
introduces more errors in the velocity field. For models with a complex geometry, the regular mesh with the element-based preprocessing method is likely to produce a "staircase" effect, which tends to increase the stress errors.

(3) We can use the Gaussian-quadrature-point based method to provide an optimal viscosity to the Gaussian quadrature point by selecting a proper sampling distance and averaging method. Increasing the sampling distance improves the stress accuracy but deteriorates the velocity accuracy. The recommended sampling radius is two-element width for $\mathrm{Q}_{1}$ elements and one-element width for $\mathrm{Q}_{2}$ elements.

(4) Regarding the averaging method of viscosity from particles to the Gauss quadrature points, the harmonic mean method is suggested for the cases where the interface is dominated by simple shear modes which produces high contrasts of strain rate across the interface.

(5) The classical superconvergent point recovery (SPR) method can be utilized to refine stress patches of interest that have been processed by combined pre- and post-processing methods, although the SPR method alone cannot effectively remove stress perturbations caused by mixed-material elements.

(6) All these preprocessing methods have a convergence rate $\left(L_{2}\right.$ norm) close to one for both the stress and velocity solutions. In contrast, the maximum stress error decays slowly with the mesh resolution and has a convergence rate far less than one, though the maximum velocity error has a convergence rate of one.

\section{Acknowledgements}

We thank Australian Research Council for funding this research under Discovery Grant DP170103350 and DP 150102887 . Testing models are run with the assistance of resources and 
services from the National Computational Infrastructure $(\mathrm{NCl})$, as well as the Pawsey Supercomputing Centre, which are supported by the Australian Governmentand the Government of Western Australia 


\section{References}

Atwater, T., Stock, J., 1998. Pacific-North America plate tectonics of the Neogene southwestern United States: an update. International Geology Review 40, 375-402.

Bardenhagen, S.G., Kober, E.M., 2004. The generalized interpolation material point method. Cmes Comp Model Eng 5, 477-495.

Bercovier, M., Pironneau, O., 1979. Error estimates for finite element method solution of the Stokes problem in the primitive variables. Numerische Mathematik 33, 211-224.

Bird, P., 2009. Long - term fault slip rates, distributed deformation rates, and forecast of seismicity in the western United States from joint fitting of community geologic, geodetic, and stress direction data sets. Journal of Geophysical Research: Solid Earth 114.

Boroomand, B., Zienkiewicz, O.C., 1997a. An improved REP recovery and the effectivity robustness test. International Journal for Numerical Methods in Engineering 40, 3247-3277.

Boroomand, B., Zienkiewicz, O.C., 1997b. Recovery by equilibrium in patches (REP). International Journal for Numerical Methods in Engineering 40, 137-164.

Braun, J., Sambridge, M., 1994. Dynamical Lagrangian Remeshing (DIr) - a New Algorithm for Solving Large-Strain Deformation Problems and Its Application to Fault-Propagation Folding. Earth and Planetary Science Letters 124, 211-220.

Braun, J., Thieulot, C., Fullsack, P., DeKool, M., Beaumont, C., Huismans, R., 2008. DOUAR: A new three-dimensional creeping flow numerical model for the solution of geological problems. Physics of the Earth and Planetary Interiors 171, 76-91.

Davies, D.R., Wilson, C.R., Kramer, S.C., 2011. Fluidity: A fully unstructured anisotropic adaptive mesh computational modeling framework for geodynamics. Geochem Geophy Geosy 12.

DeMets, C., Dixon, T.H., 1999. New kinematic models for Pacific-North America motion from $3 \mathrm{Ma}$ to present, I: Evidence for steady motion and biases in the NUVEL-1A model. Geophysical Research Letters 26, 1921-1924.

Deubelbeiss, Y., Kaus, B.J.P., 2008. Comparison of Eulerian and Lagrangian numerical techniques for the Stokes equations in the presence of strongly varying viscosity. Physics of the Earth and Planetary Interiors 171, 92-111.

Duretz, T., May, D.A., Gerya, T.V., Tackley, P.J., 2011. Discretization errors and free surface stabilization in the finite difference and marker - in - cell method for applied geodynamics: A numerical study. Geochemistry, Geophysics, Geosystems 12.

Gassmöller, R., Lokavarapu, H., Bangerth, W., Puckett, E.G., 2019. Evaluating the accuracy of hybrid finite element/particle-in-cell methods for modelling incompressible Stokes flow. Geophysical Journal International 219, 1915-1938. 
Gerya, T.V., Yuen, D.A., 2003. Rayleigh-Taylor instabilities from hydration and melting propel 'cold plumes' at subduction zones. Earth and Planetary Science Letters 212, 47-62.

Harlow, F.H., 1964. The particle-in-cell computing method for fluid dynamics. Methods Comput. Phys. 3, 319-343.

Hinton, E., Campbell, J.S., 1974. Local and global smoothing of discontinuous finite element functions using a least squares method. International Journal for Numerical Methods in Engineering $8,461-480$.

Hughes, T.J.R., 2012. The finite element method: linear static and dynamic finite element analysis. Courier Corporation.

Lenardic, A., Moresi, L.N., Mühlhaus, H., 2003. Longevity and stability of cratonic lithosphere: Insights from numerical simulations of coupled mantle convection and continental tectonics. Journal of Geophysical Research: Solid Earth 108, n/a-n/a.

Maddison, J.R., Hiester, H.R., 2017. Optimal Constrained Interpolation in Mesh-Adaptive Finite Element Modeling. Siam Journal on Scientific Computing 39, A2257-A2286.

Mansour, J., Giordani, J., Moresi, L., Beucher, R., Kaluza, O., Velic, M., Farrington, R.J., Quenette, S., Beall, A.P., 2020. Underworld2: Python geodynamics modelling for desktop, hpc and cloud. Journal of Open Source Software 5, 1797.

May, D.A., 2009. The implicit material point method for variable viscosity stokes flow, School of Mathematical Sciences. Monash University.

May, D.A., Moresi, L., 2008. Preconditioned iterative methods for Stokes flow problems arising in computational geodynamics. Physics of the Earth and Planetary Interiors 171, 33-47.

Meade, B.J., Hager, B.H., 2005. Block models of crustal motion in southern California constrained by GPS measurements. J Geophys Res-Sol Ea 110.

Moresi, L., Dufour, F., Muhlhaus, H.B., 2002. Mantle convection modeling with viscoelastic/brittle lithosphere: Numerical methodology and plate tectonic modeling. Pure and Applied Geophysics 159, 2335-2356.

Moresi, L., Quenette, S., Lemiale, V., Meriaux, C., Appelbe, B., Muhlhaus, H.B., 2007. Computational approaches to studying non-linear dynamics of the crust and mantle. Physics of the Earth and Planetary Interiors 163, 69-82.

Moresi, L., Zhong, S., Gurnis, M., 1996. The accuracy of finite element solutions of Stokes's flow with strongly varying viscosity. Physics of the Earth and Planetary Interiors 97, 83-94.

Poliakov, A., Podladchikov, Y., 1992. Diapirism and Topography. Geophysical Journal International 109, 553-564.

Puckett, E.G., Turcotte, D.L., He, Y., Lokavarapu, H., Robey, J.M., Kellogg, L.H., 2018. New numerical approaches for modeling thermochemical convection in a compositionally stratified fluid. Physics of the Earth and Planetary Interiors 276, 10-35.

Ramière, I., 2008. Convergence analysis of the Q1 - finite element method for elliptic problems with non - boundary - fitted meshes. International Journal for Numerical Methods in Engineering $75,1007-1052$. 
Ranalli, G., 1995. Rheology of the Earth. Springer Science \& Business Media.

Schmeling, H., Babeyko, A.Y., Enns, A., Faccenna, C., Funiciello, F., Gerya, T., Golabek, G.J., Grigull, S., Kaus, B.J.P., Morra, G., 2008. A benchmark comparison of spontaneous subduction models-Towards a free surface. Physics of the Earth and Planetary Interiors 171, 198-223.

Sharples, W., Moresi, L.N., Velic, M., Jadamec, M.A., May, D.A., 2016. Simulating faults and plate boundaries with a transversely isotropic plasticity model. Physics of the Earth and Planetary Interiors 252, 77-90.

Sulsky, D., Chen, Z., Schreyer, H.L., 1994. A Particle Method for History-Dependent Materials. Computer Methods in Applied Mechanics and Engineering 118, 179-196.

Taylor, C., Hood, P., 1973. A numerical solution of the Navier-Stokes equations using the finite element technique. Computers \& Fluids 1, 73-100.

Thielmann, M., Kaus, B., 2012. Shear heating induced lithospheric-scale localization: Does it result in subduction? Earth and Planetary Science Letters 359, 1-13.

Thielmann, M., May, D.A., Kaus, B.J.P., 2014. Discretization Errors in the Hybrid Finite Element Particle-in-cell Method. Pure and Applied Geophysics 171, 2165-2184.

Wallstedt, P.C., Guilkey, J.E., 2011. A weighted least squares particle - in - cell method for solid mechanics. International journal for numerical methods in engineering 85, 1687-1704.

Xia, D.F., Xu, S.L., Qi, F., 1999. A proof of the arithmetic mean-geometric mean-harmonic mean inequalities. RGMIA research report collection 2.

Zhong, S., 1996. Analytic solutions for Stokes' flow with lateral variations in viscosity. Geophysical Journal International 124, 18-28.

Zienkiewicz, O.C., Zhu, J.Z., 1992a. The superconvergent patch recovery and a posteriori error estimates. Part 1: The recovery technique. International Journal for Numerical Methods in Engineering 33, 1331-1364.

Zienkiewicz, O.C., Zhu, J.Z., 1992b. The superconvergent patch recovery and a posteriori error estimates. Part 2: Error estimates and adaptivity. International Journal for Numerical Methods in Engineering 33, 1365-1382. 


\section{Figures}

(a) Node-based method

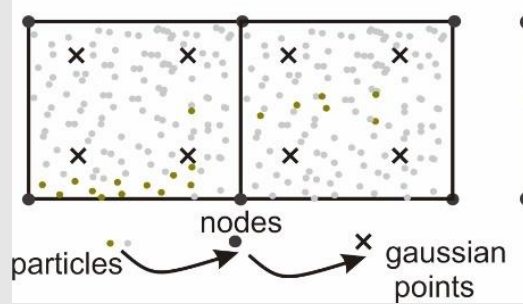

(b) Element-based method

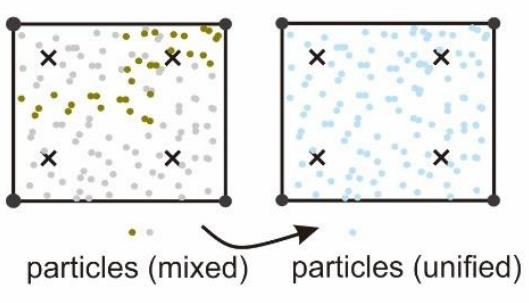

(c) Gaussian-point-based method

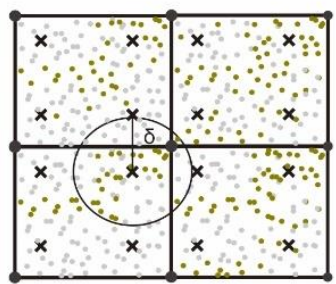

Figure 1. Sketches illustrating three preprocessing methods. (a) The node-based method first projects reciprocals of particle viscosities to the mesh nodes, and then interpolate the reciprocals of node values to the Gaussian quadrature points. (b) The element-based method first locates the cells that contain different viscosities, then replaces the viscosities in the mixed-material cells with harmonic mean value of the materials involved. (c) The Gaussian-quadrature-point based method directly projects those particles that are within a distance of $\delta$ to the Gaussian point. 


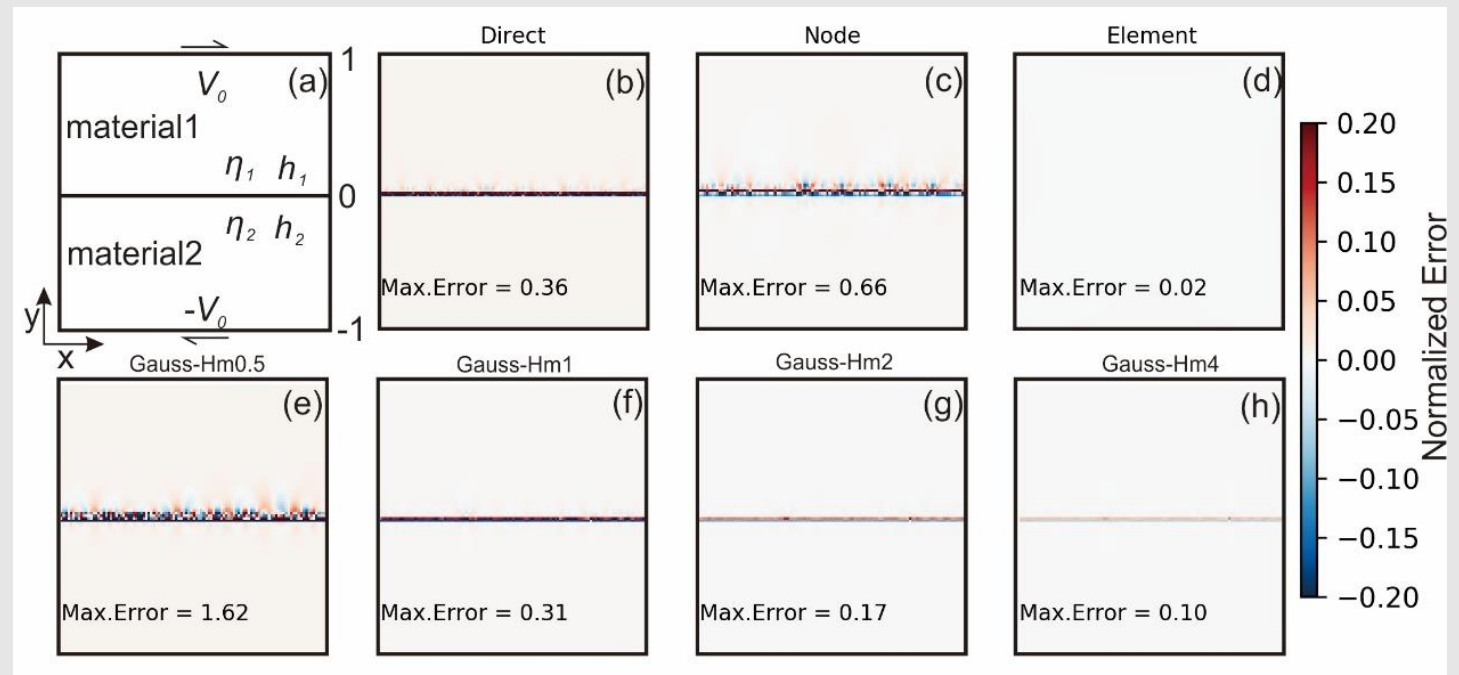

Figure 2. Simple shear model setup (a) and results of shear stress for the Direct sampling method (b), the node-based method (c), the element-based method (d), the Gaussian-quadrature-point based method with harmonic mean that averages particles within a distance of half element size to the corresponding Gaussian point (Gauss-Hm0.5) (e), the Gauss-Hm1 method (f), the Gauss$\mathrm{Hm} 2$ method $(\mathrm{g})$ and the Gauss-Hm4 method $(\mathrm{h})$. The color bar shows the normalized error. The mesh consists of $133 \times 133$ regular $\mathrm{Q}_{1}$ elements with $\alpha=0.5$ (interface at $\mathrm{y}=0$ ) 

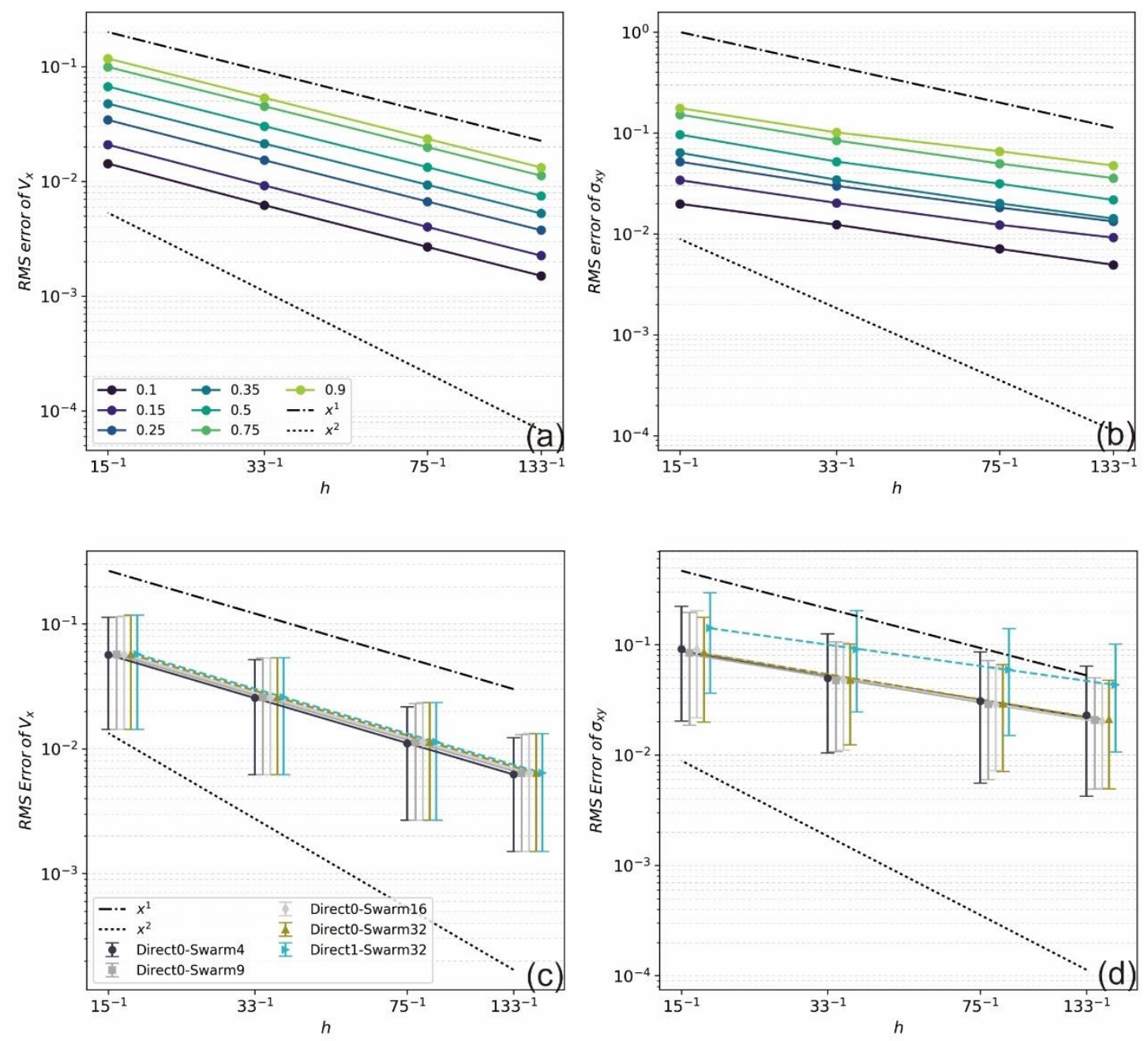

Figure 3. The RMS errors for $V_{x}(\mathrm{a})$ and $\sigma_{x y}(\mathrm{~b})$ with ensembles that have the intra-element viscosity discontinuity at different levels of the mixed-material element. The effect of particle numbers and post-processing methods on the RMS error is compared in (c) and (d). Direct0 represents the case that does not have any preprocessing procedure but has used the postprocessing method averaging around local nodes with shape functions and the Direct1 uses the global projection method with least square fits. The case in (a) and (b) corresponds to the Direct0-Swarm32 which has 32 particles per cell and is postprocessed with the Direct0 method. Solid lines in (a) and (b) represent different $\alpha$ values while the bounding dash-dot and dashed 
lines shows convergence rate of 1 and 2, respectively. The caps of the vertical line denote the upper and lower limit of the ensembles. The mean values are represented by different markers. We fit the mean value of the ensembles versus the mesh size by linear regression. The slope of the regression line is the convergence rate. For tests with the same mesh resolution, each case is shifted a little bit horizontally to de-collapse the vertical bars. The plotting rules are applied to all other convergence rate studies listed below. 

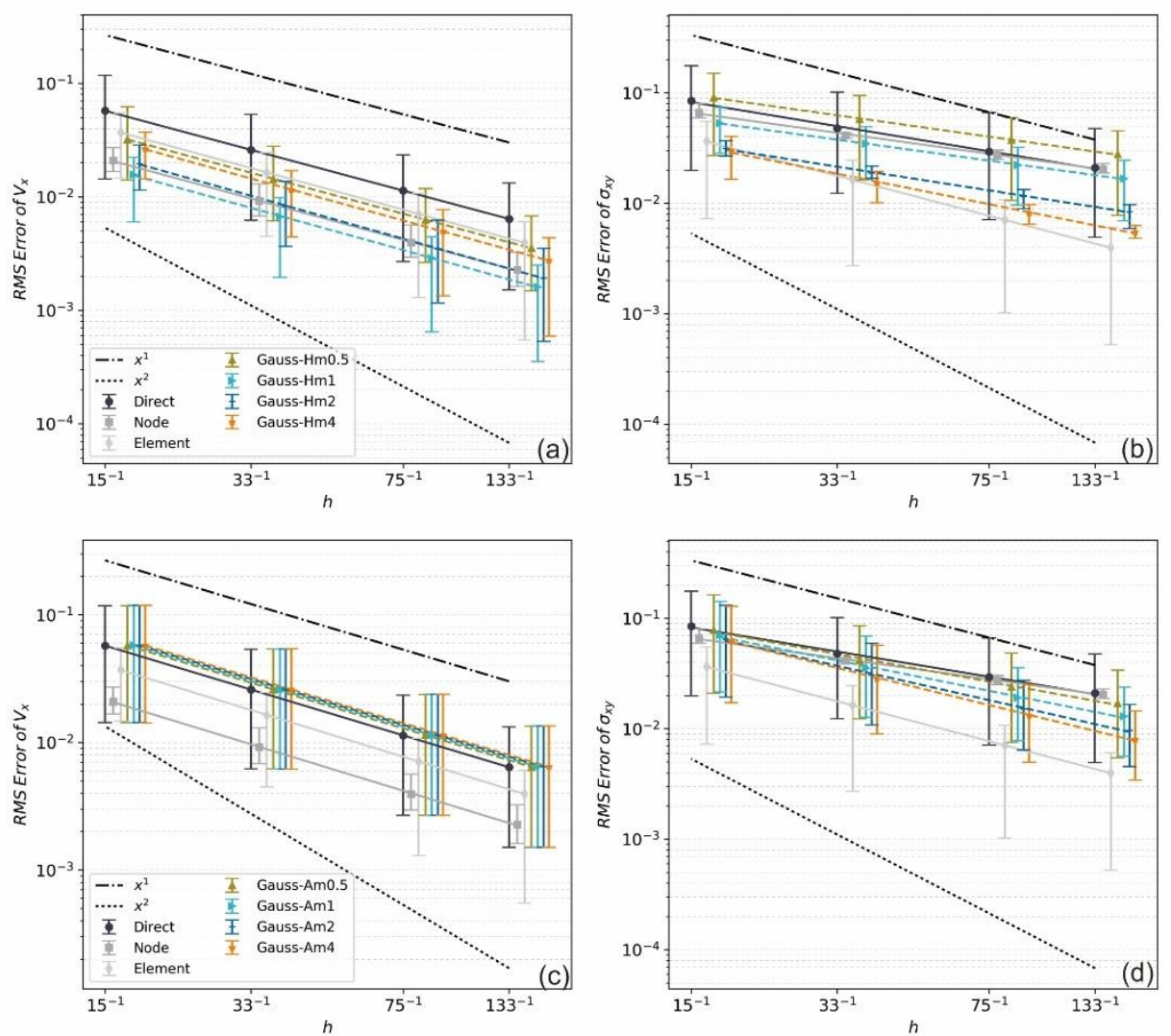

Figure 4. The $V_{x}(\mathrm{a} \& \mathrm{c})$ and $\sigma_{x y}(\mathrm{~b} \& \mathrm{~d}) \mathrm{RMS}$ error calculated with $\mathrm{Q}_{1}$ elements for different preprocessing methods applied to the simple shear model with regular mesh. Every row shares the same legend. The solutions calculated with the Direct method, node-based method and Element based method are plot in both the upper and lower panels as a refence to be compared with the Gauss-Hm method in the upper panel (a \& b) and the Gauss-Am method in lower panel (c \& d). 

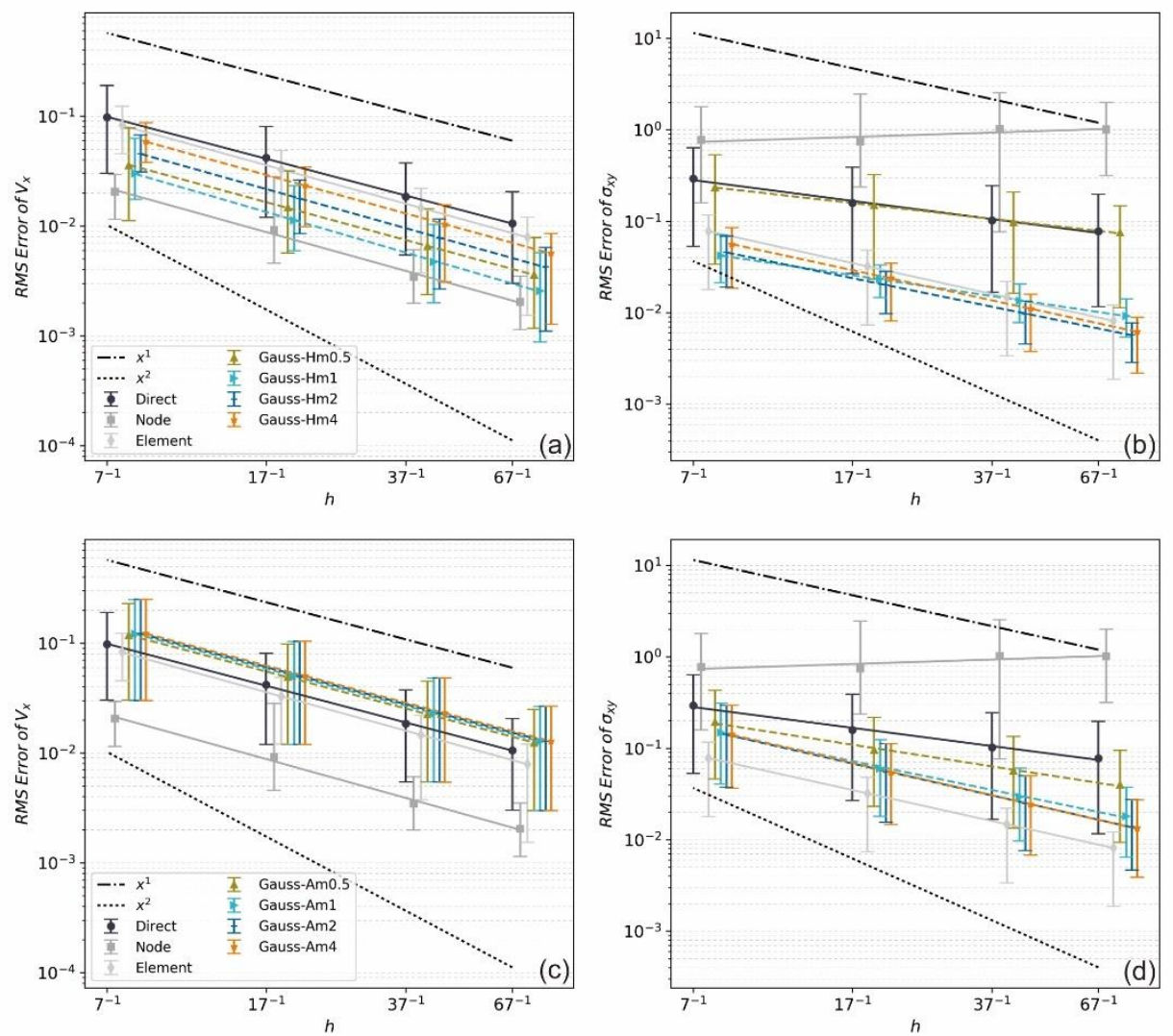

Figure 5. The $V_{x}(\mathrm{a} \& \mathrm{c})$ and $\sigma_{x y}(\mathrm{~b} \& \mathrm{~d}) \mathrm{RMS}$ error calculated with $\mathrm{Q}_{2}$ elements for different preprocessing methods applied to the simple shear model with regular mesh. Every row shares the same legend. 


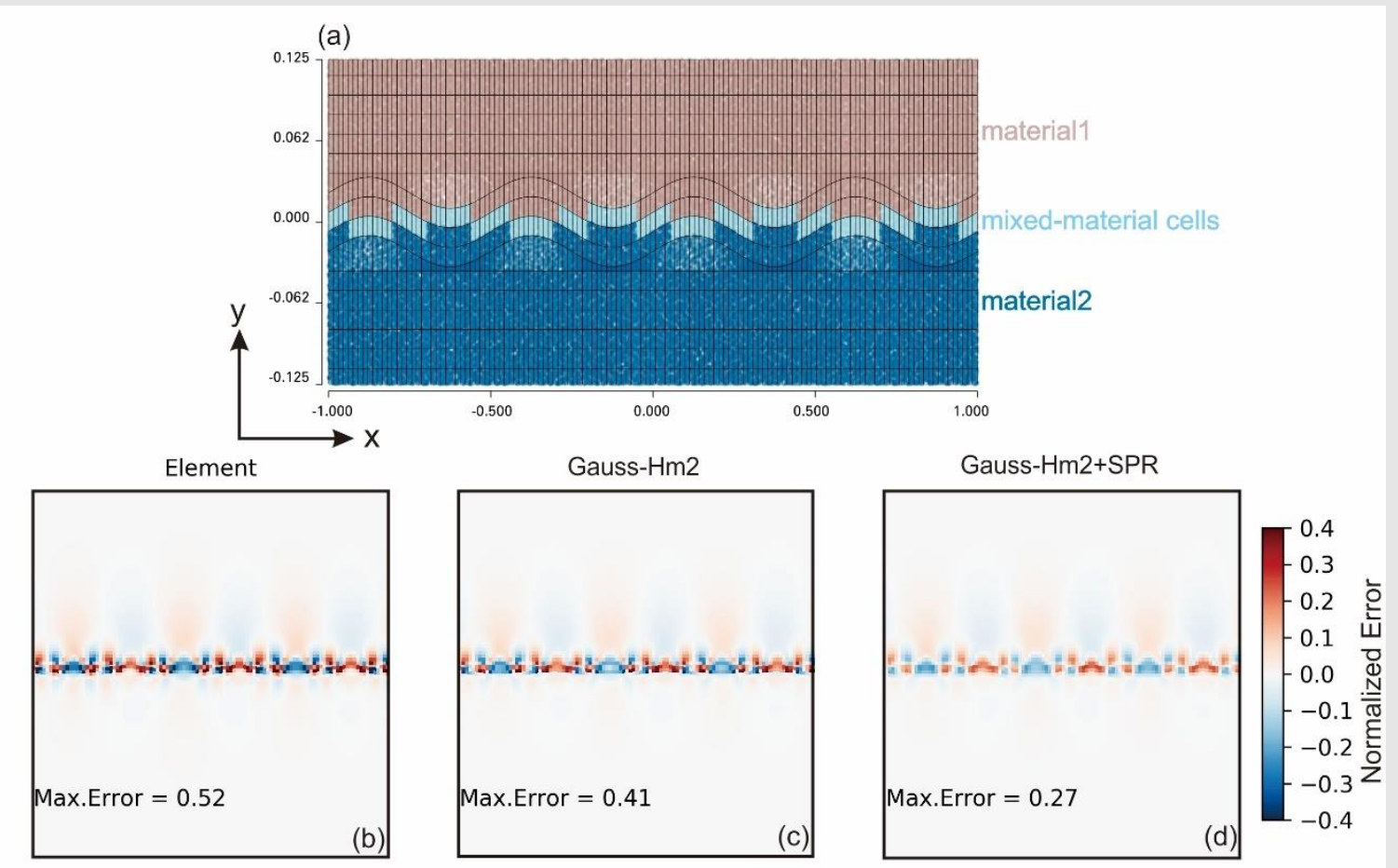

Figure 6. Simple shear model setup with distorted mesh surrounding the interface with $\beta=$ 0.8 (a) and results of shear stress for the element-based method (b), the Gauss-Hm2 method (c), and post-processing with SPR on Gauss-Hm2 results (d). The color bar shows the normalized error. The mesh consists of $133 \times 133$ regular $\mathrm{Q}_{1}$ elements. The model setup shows material distribution after being preprocessed with the element-based method, and the mesh in $y$ direction is exaggerated by 4 to see the details of deformed mesh. 

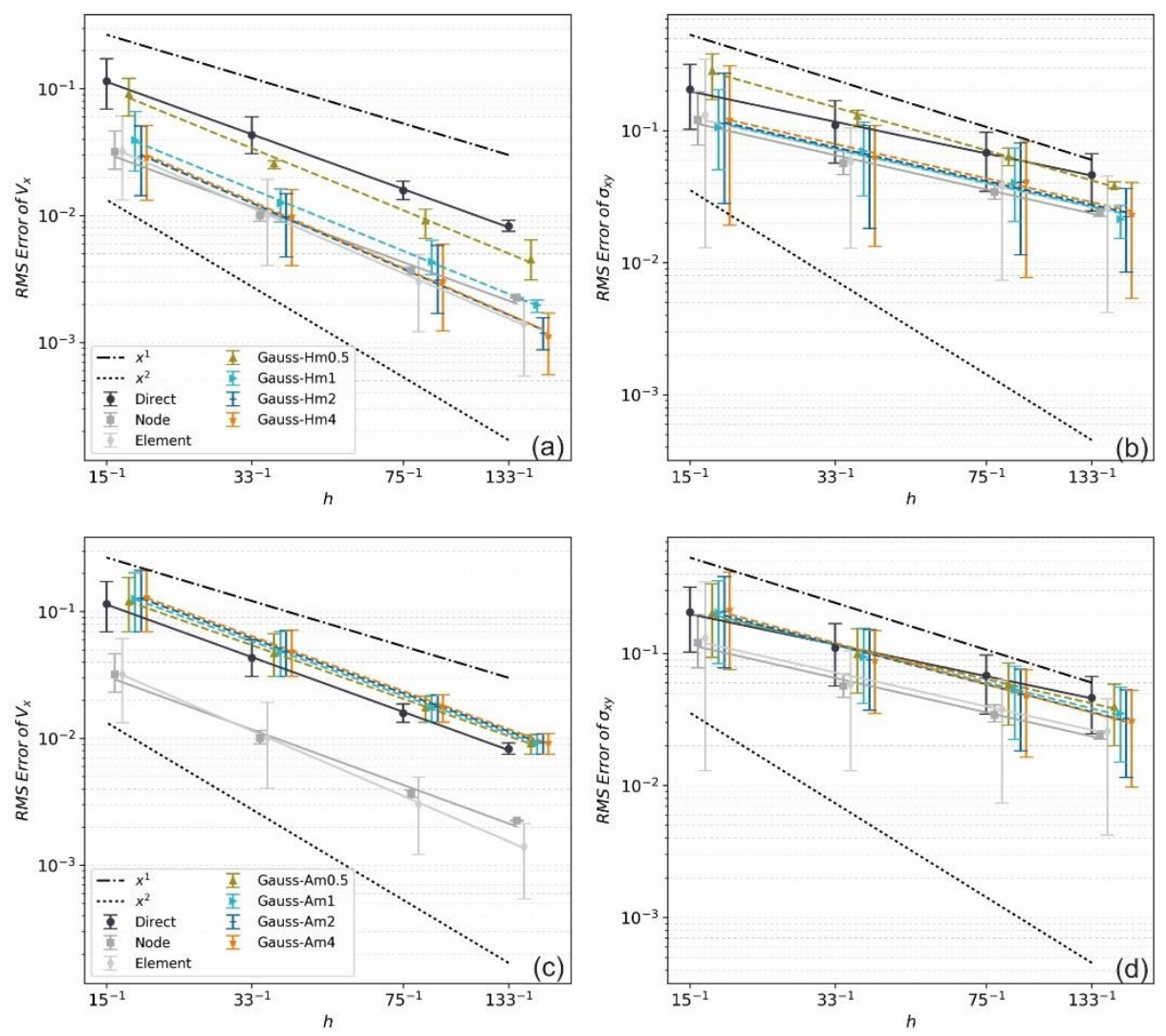

Figure 7. The $V_{x}(a \& c)$ and $\sigma_{x y}(b \& d)$ RMS error calculated with $\mathrm{Q}_{1}$ elements for different preprocessing methods applied to the simple shear model with distorted mesh. Every row shares the same legend. The ensembles area created with different $\beta$. 

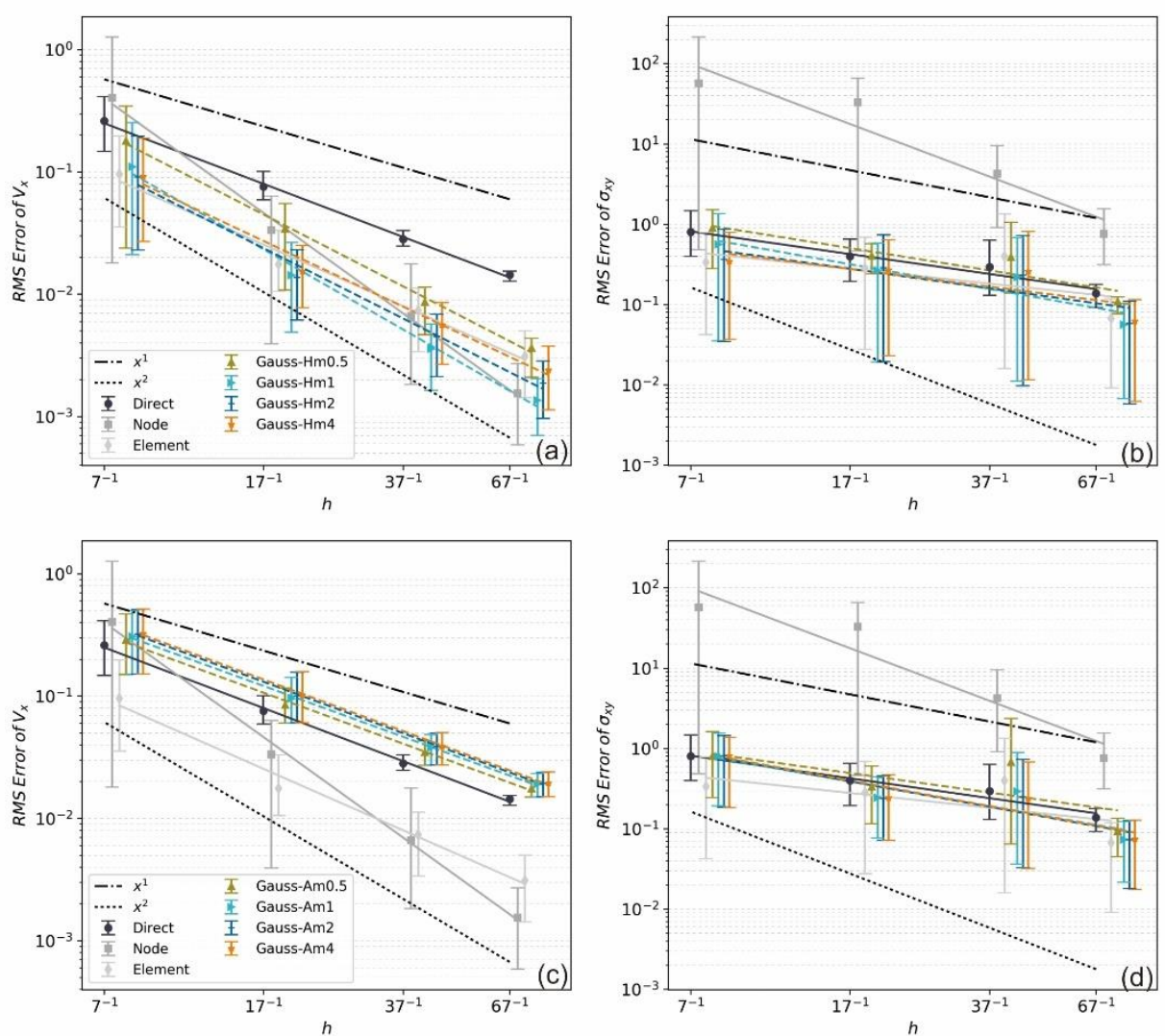

Figure 8. The $V_{x}(\mathrm{a} \& \mathrm{c})$ and $\sigma_{x y}(\mathrm{~b} \& \mathrm{~d}) \mathrm{RMS}$ error calculated with $\mathrm{Q}_{2}$ elements for different preprocessing methods applied to the simple shear model with distorted mesh. Every row shares the same legend. 
(a)

$\log _{10}$ (Viscosity)

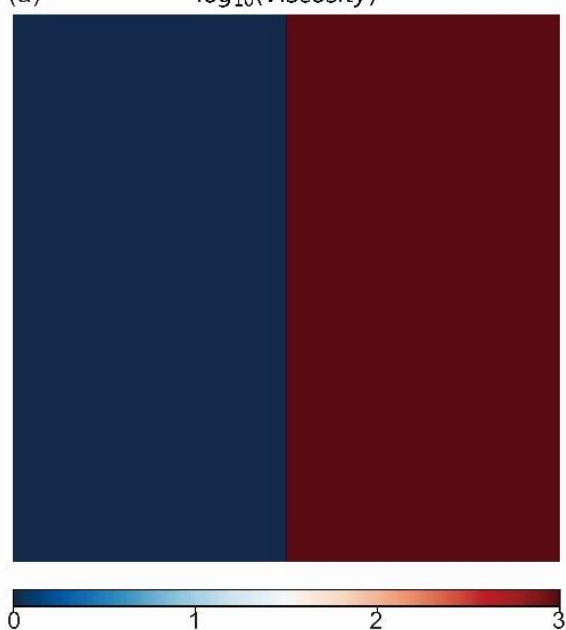

(b)

Density

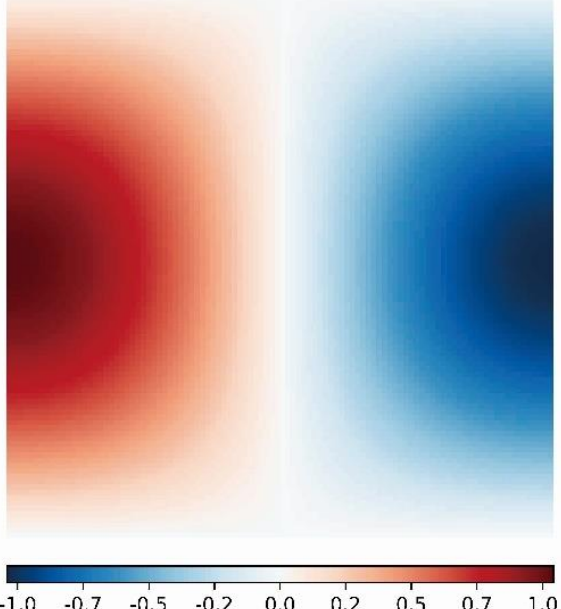

Figure 9. Model setup for the SolCx benchmark. 

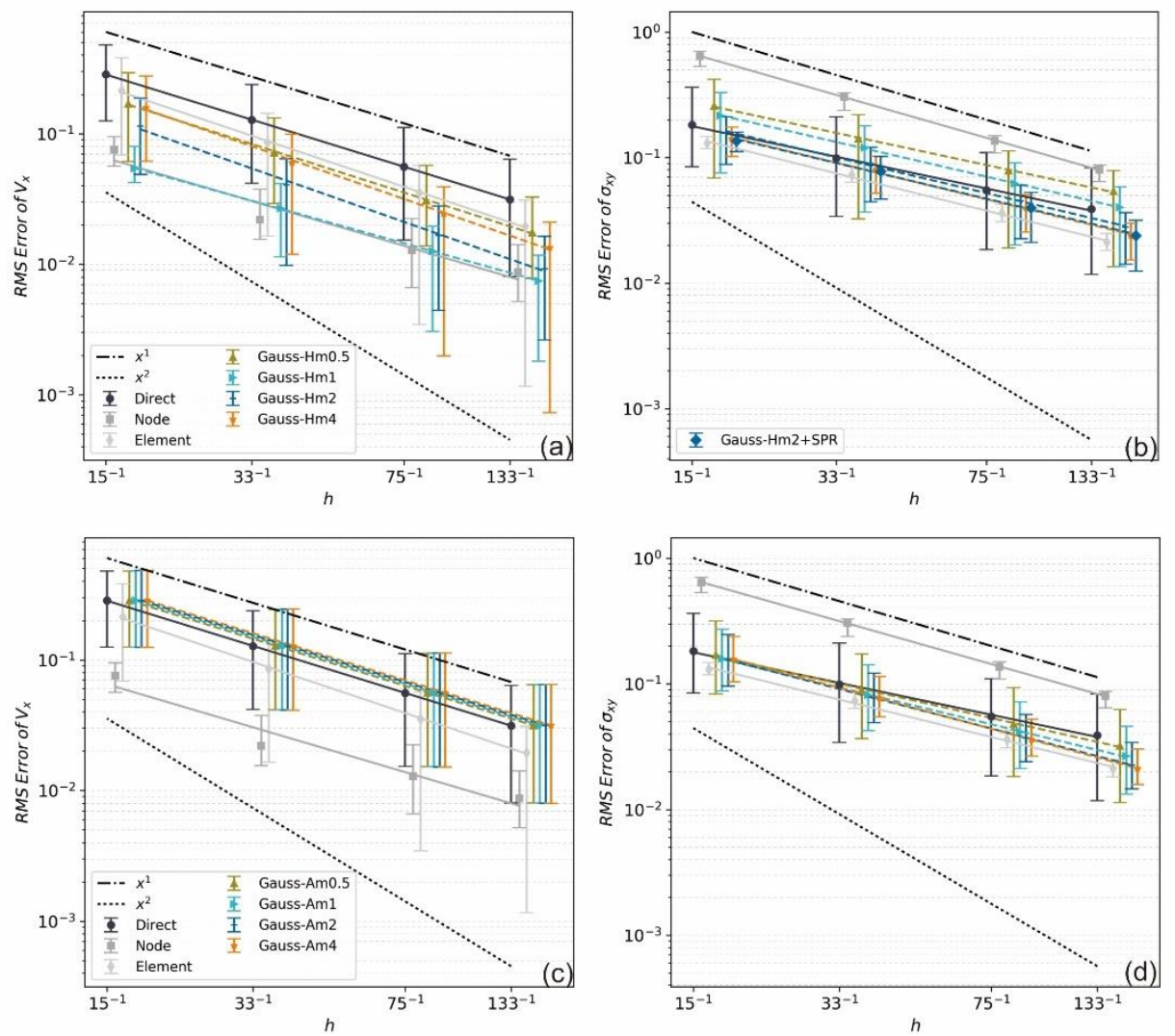

Figure 10. The $V_{x}(\mathrm{a} \& \mathrm{c})$ and $\sigma_{x y}(\mathrm{~b} \& \mathrm{~d}) \mathrm{RMS}$ error calculated with $\mathrm{Q}_{1}$ elements for different preprocessing methods applied to the SolCx benchmark model. Every row shares the same legend. 

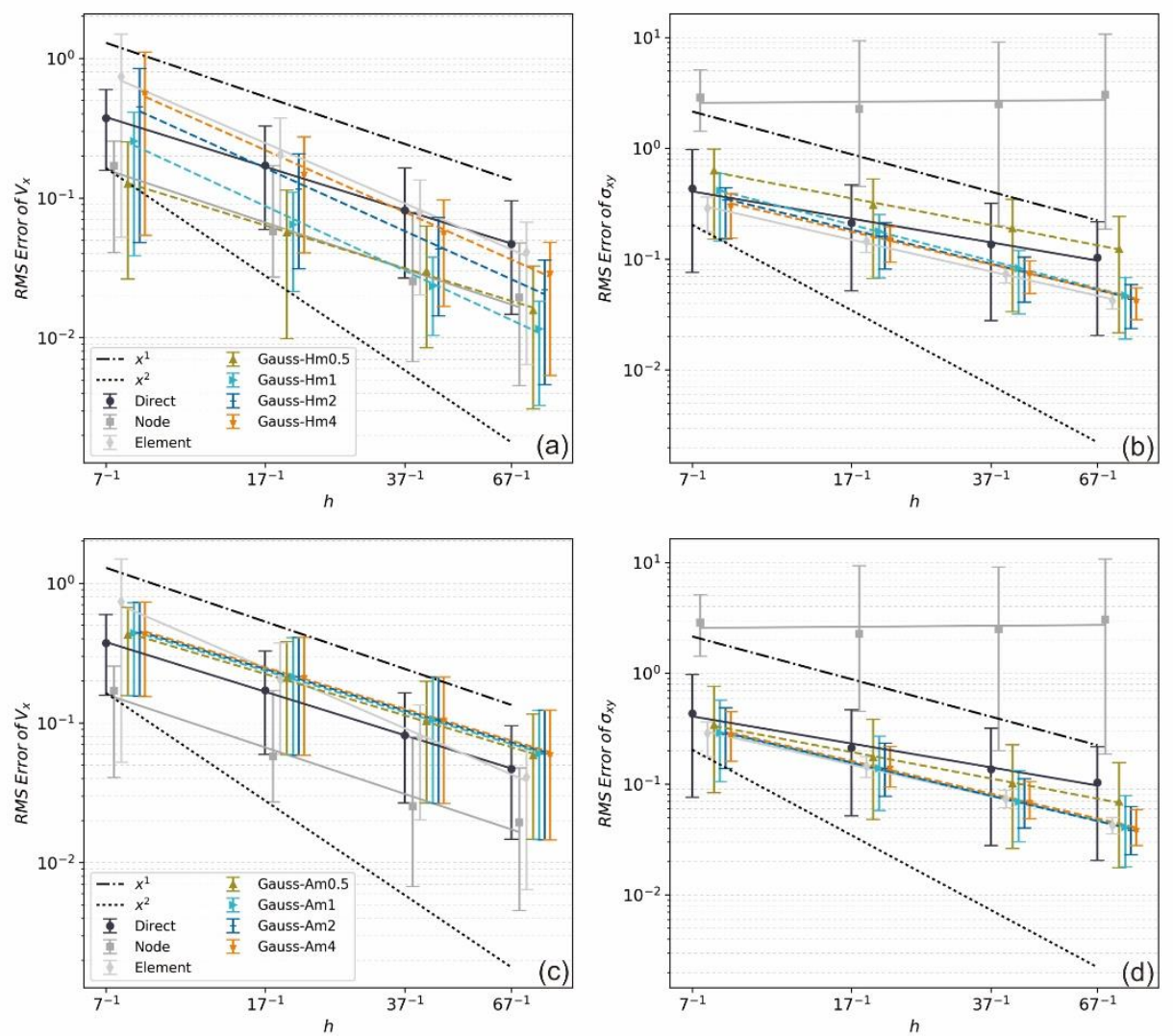

Figure 11. The $V_{x}(a \& c)$ and $\sigma_{x y}(b \& d)$ RMS error calculated with $\mathrm{Q}_{2}$ elements for different preprocessing methods applied to the SolCx benchmark model. Every row shares the same legend. 

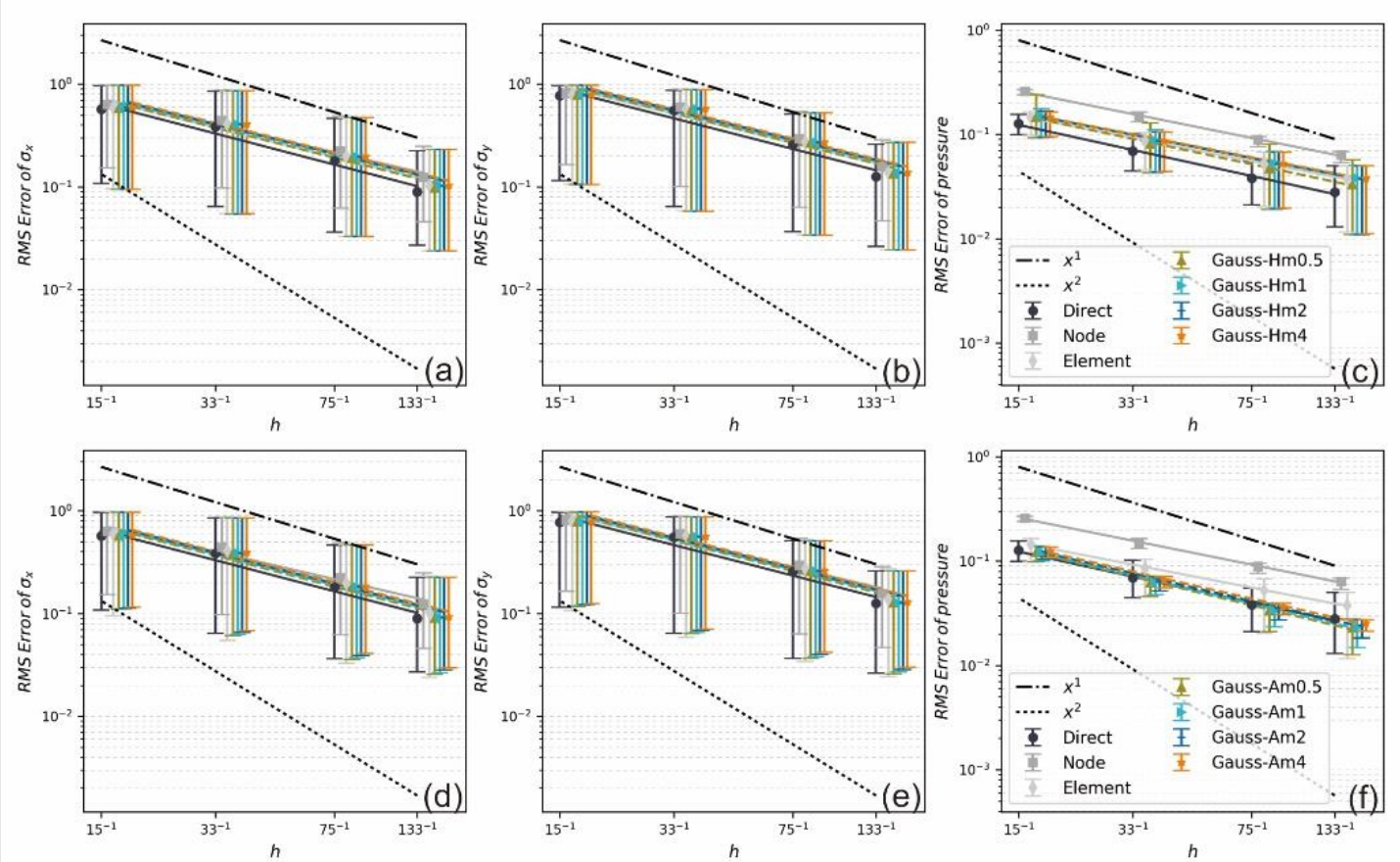

Figure 12. The $\sigma_{x}(\mathrm{a} \& \mathrm{~d}), \sigma_{y}(\mathrm{~b} \& \mathrm{e})$ and pressure $(\mathrm{c} \& \mathrm{f}) \mathrm{RMS}$ error calculated with $\mathrm{Q}_{1}$ elements for different preprocessing methods applied to the SolCx benchmark model. Every row shares the same legend. 

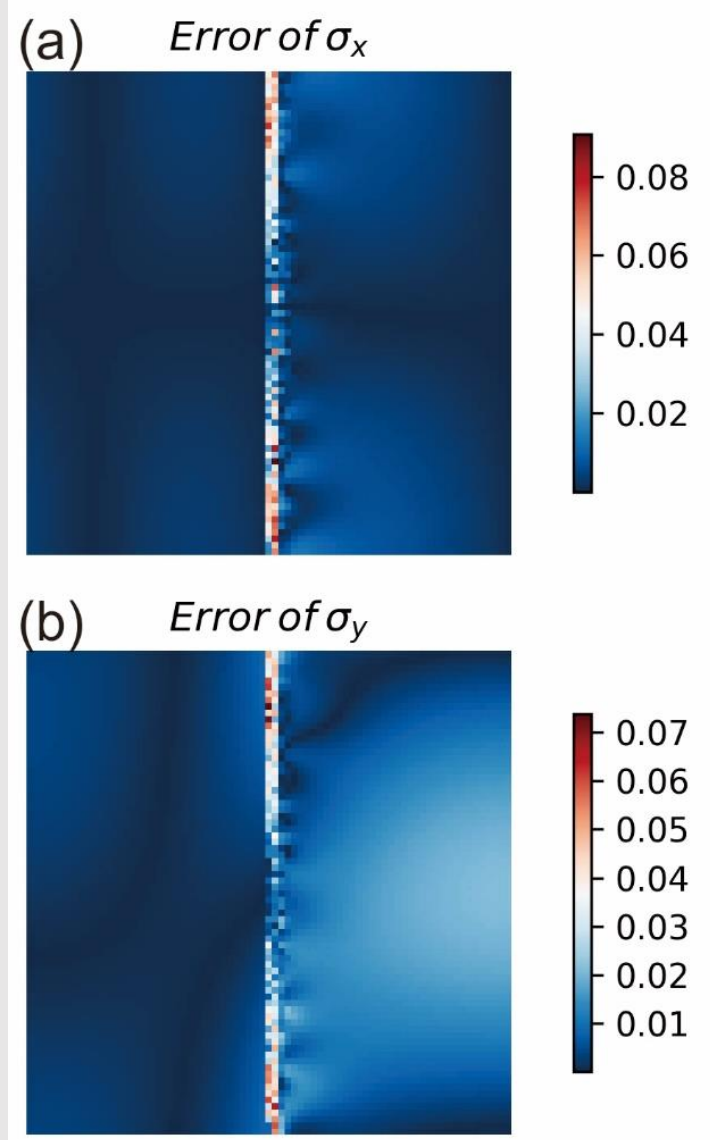

(c) Error of $\sigma_{x y}$

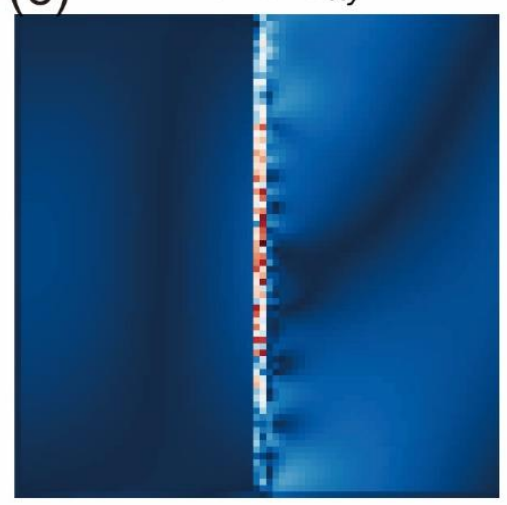

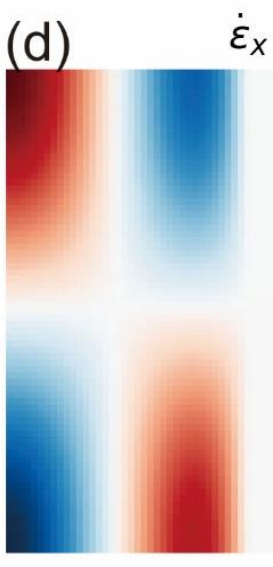

$\dot{\varepsilon}_{x}$
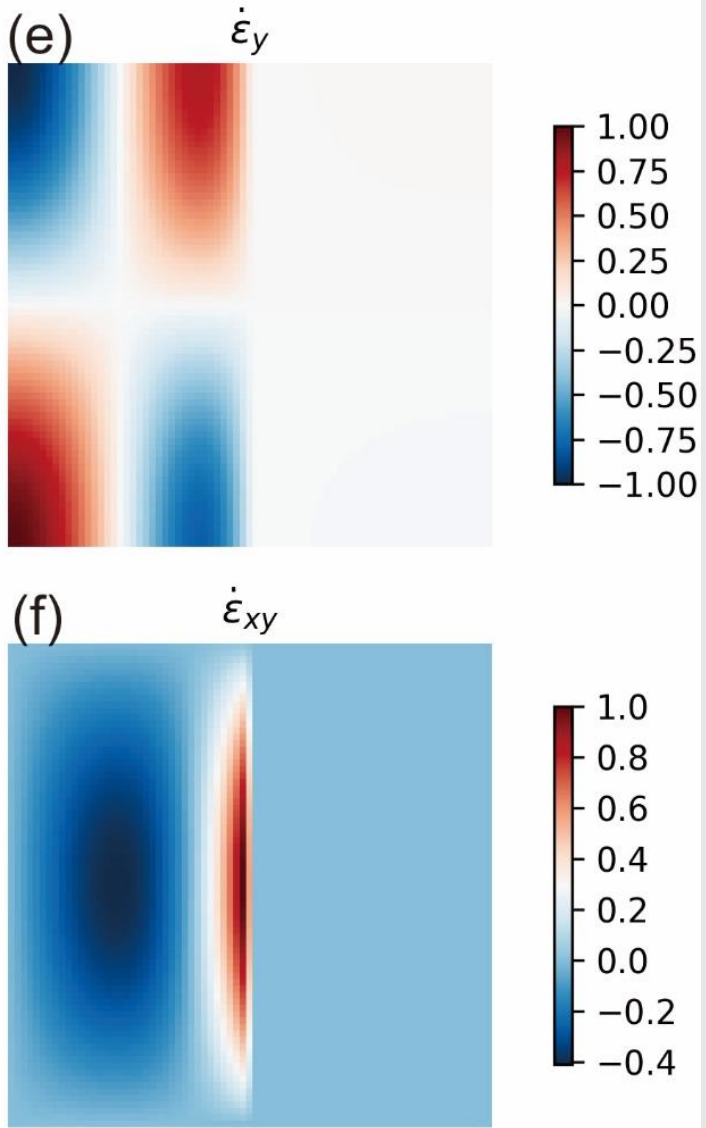

Figure 13. The normalized absolute error for $\sigma_{x}(\mathrm{a}), \sigma_{y}(\mathrm{~b})$ and $\sigma_{x y}(\mathrm{c})$, and the analytical solution for strain rate, $\dot{\varepsilon}_{x}(\mathrm{~d}), \dot{\varepsilon}_{y}(\mathrm{e})$ and $\dot{\varepsilon}_{x y}$ (f). The stress error is normalized by the maximum value of the corresponding analytical solution for each component. The strain rate is normalized by the maximum value for each component. The stress error is calculated with $75 \times 75$ regular $\mathrm{Q}_{1}$ elements. 

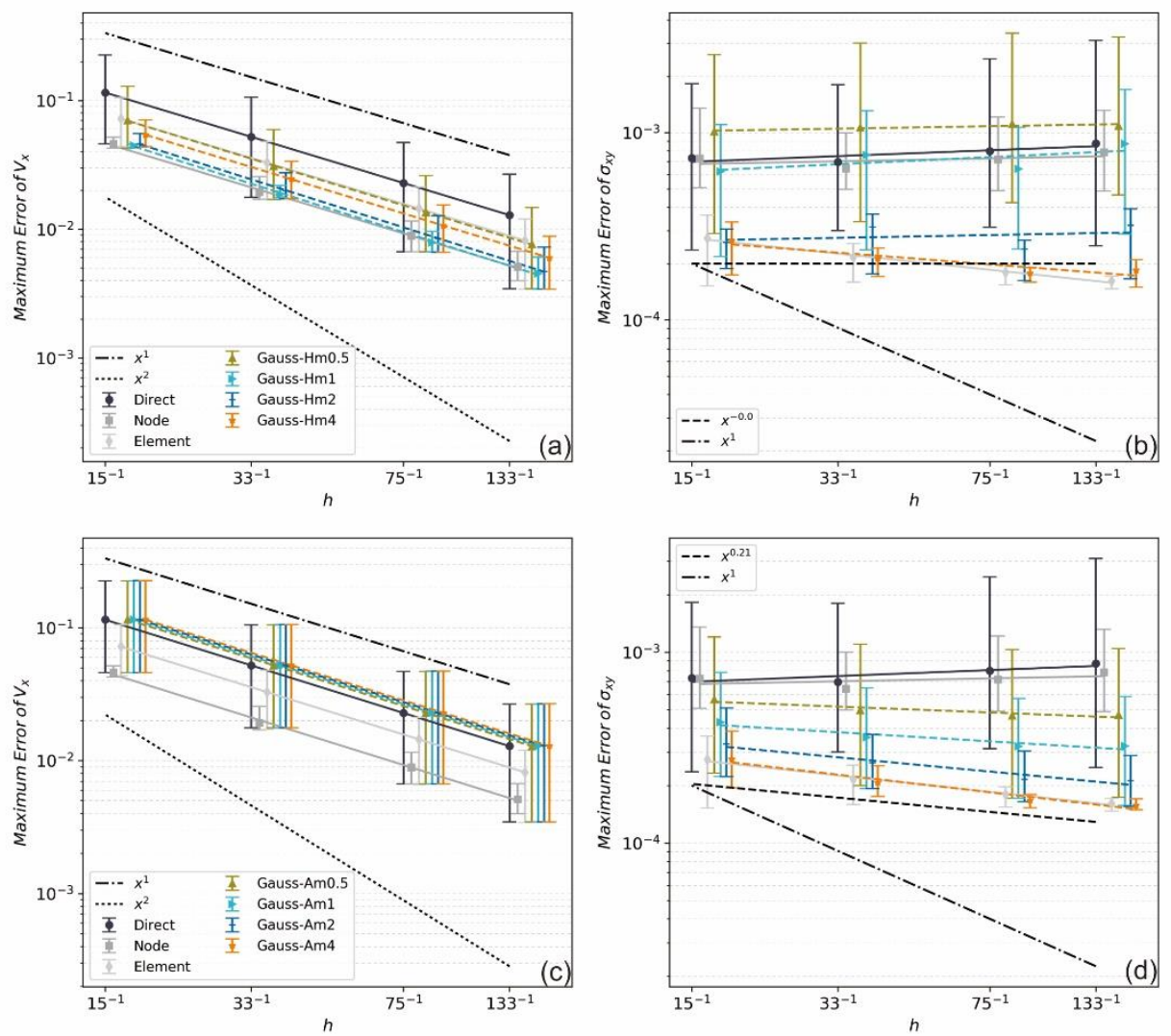

Figure 14. The $V x(a \& c)$ and $\sigma_{x y}(b \& d)$ maximum error calculated with $\mathrm{Q} 1$ elements for different preprocessing methods applied to the simple shear model with regular mesh. Every row shares the same legend. The black dashed line in the right panel $(b \& d)$ has the same slope of the Gaussian-quadrature-point based method with a sampling radius of two element size. 




Figure 15. Models with an embedded weak zone (dark blue particles at $x=-0.21-0.21$ ) that is at a low angle $\left(\sim 10^{\circ}\right)$ to the $x$-axis. The model size is $80 \mathrm{~km} \times 20 \mathrm{~km}$, and the length shown here is normalized by $100 \mathrm{~km}$. $\mathrm{Q}_{1}$ elements of $300 \times 150$ resolution is applied. The mesh edges in (a) are not aligned with the weak zone strike, while that in (b) is designed to be aligned with material interfaces, and the mesh in the central part that is close to the weak zone is refined in $y$ direction as well (b). That means material mixing happens in (a) but not in (b). Note that the meshes in (a) and (b) are zoomed to see details of the mesh structure and only part of the 
calculation domain is shown. The whole-domain shear stress of the case in (b) is shown in (c).

The results of the case in (a) are illustrated in Figure 16. 

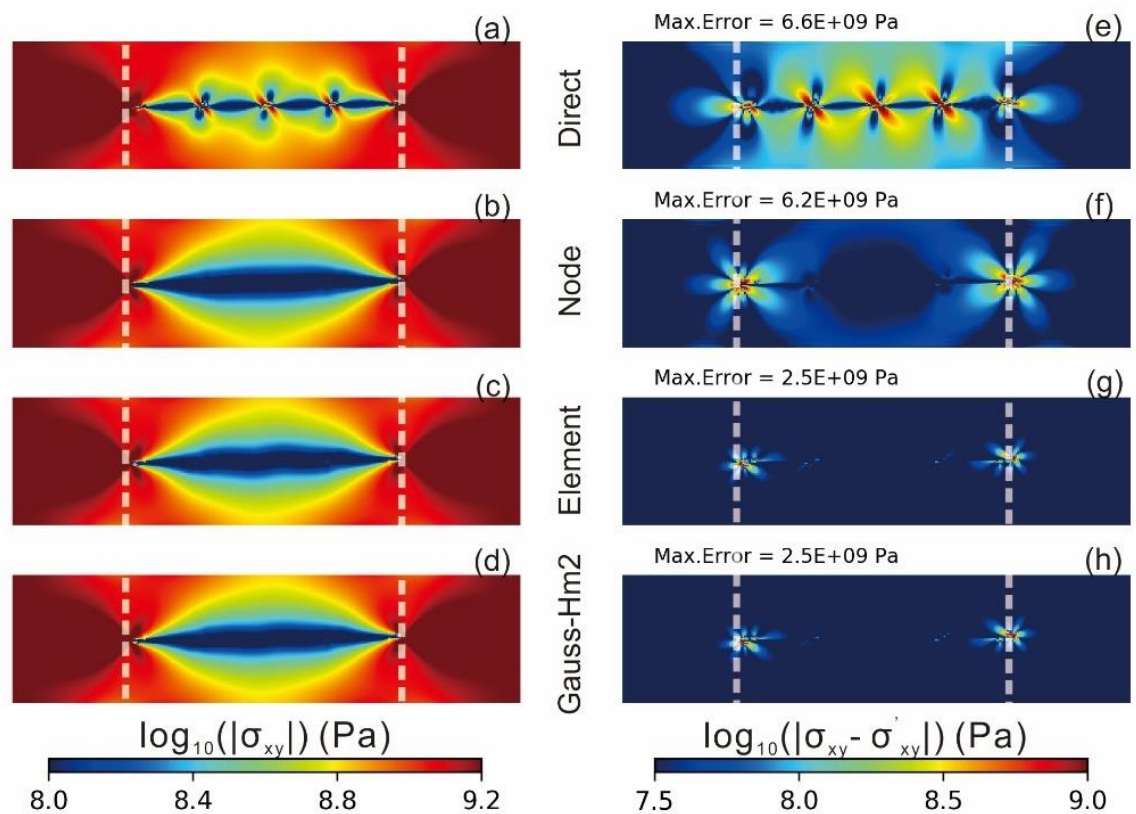

Figure 16. The results based on the mesh in Figure 15a which has mixing materials in elements.

The left panel (a-d) shows the absolute shear stress, and the right panel (e-h) shows the difference with respect to the reference model for different pre-processing methods. $\sigma_{x y}^{\prime}$ is the stress in the reference model. The white dashed line marks the end of fault tips. 


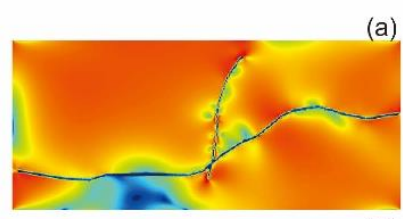

(b)

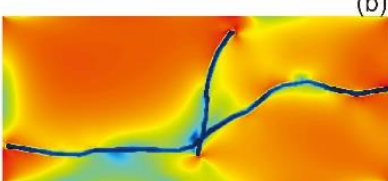

(c)

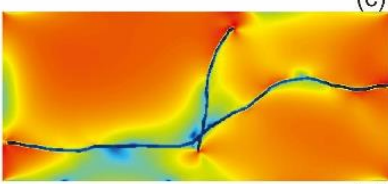

(d)

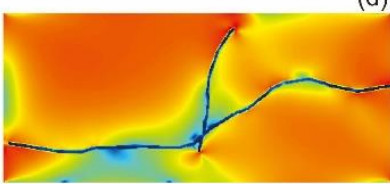

(e)
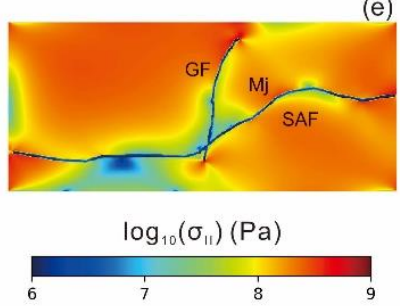
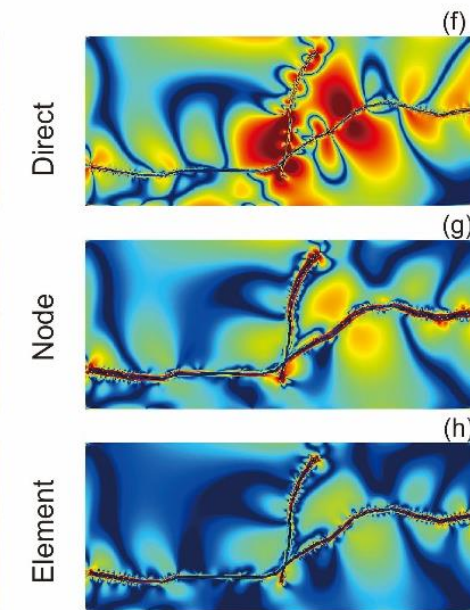

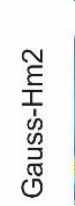
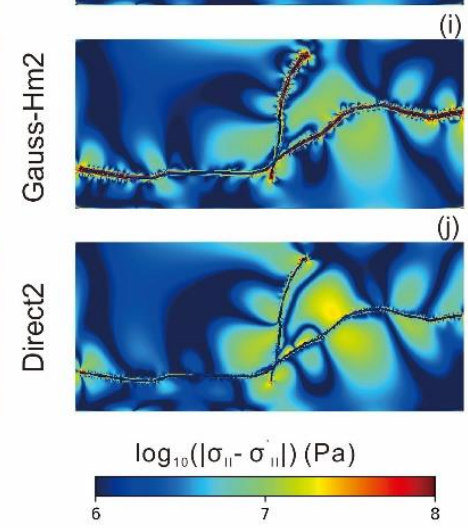
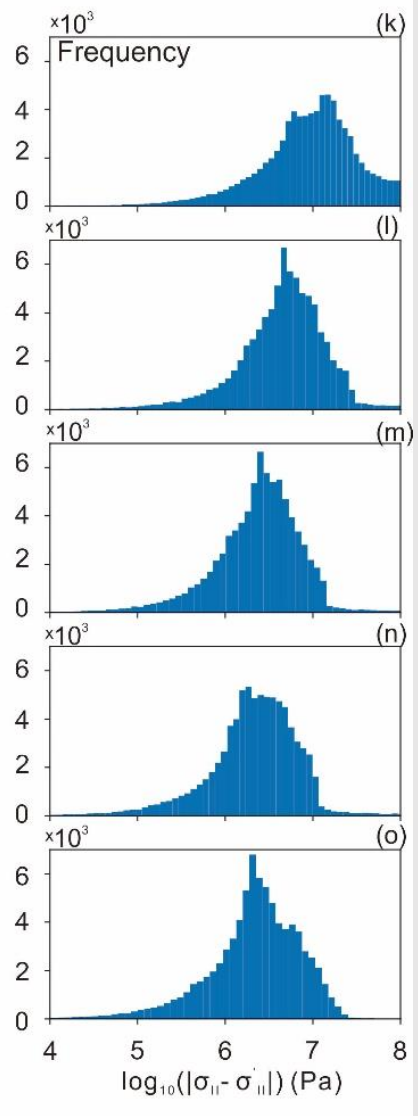

Figure 17. The second invariant stress of the synthetic model with complex fault geometries that are derived from the San Andreas Fault system in California (Bird, 2009). The left panel (ae) shows absolute shear stress, the middle panel ( $f-j)$ shows the spatial distribution of the difference with respect to the reference model for different pre-processing methods and the right panel $(\mathrm{k}-\mathrm{o})$ demonstrate the histogram of the errors for each case. $\sigma_{I I}^{\prime}$ is the stress in the reference model, which has four times resolution of models processed with the Direct, nodebased, element-based, and Gauss-Hm2 method. With others being equal, the Direct2 models has twice resolution of the Direct model, which is calculated with $400 \times 200 \mathrm{Q}_{1}$ elements. Briefs for major faults and blocks: SAF - San Andreas Fault, GF - Garlock Fault, Mj - Mojave block. 


\section{Appendix}
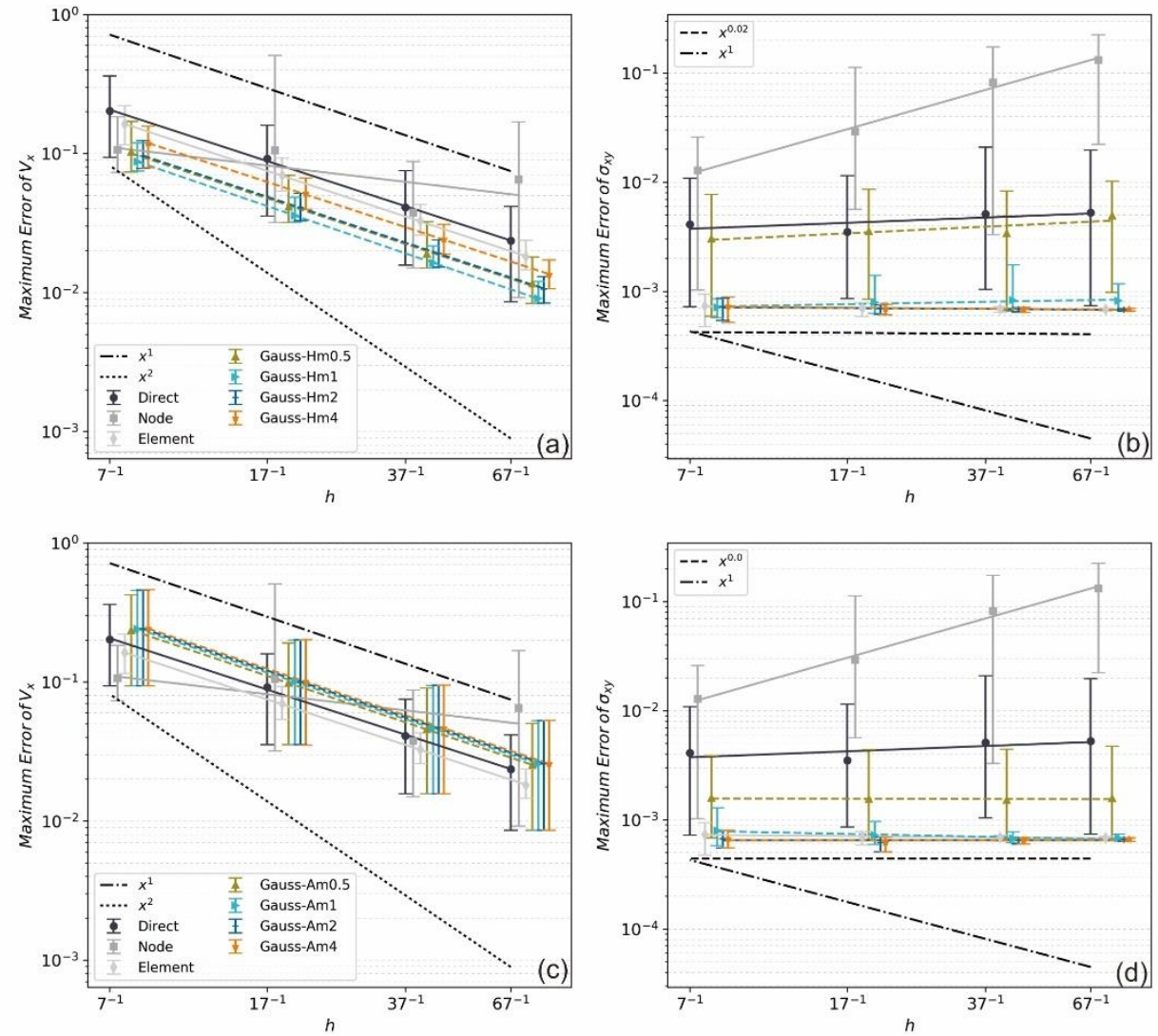

Figure (A1). The $V_{x}(\mathrm{a} \& \mathrm{c})$ and $\sigma_{x y}(\mathrm{~b} \& \mathrm{~d})$ maximum error calculated with $\mathrm{Q}_{2}$ elements for different preprocessing methods applied to the simple shear model with regular mesh. Every row shares the same legend. The black dashed line in the right panel $(b \& d)$ has the same slope of the Gaussian-quadrature-point based method with a sampling radius of one element size. 

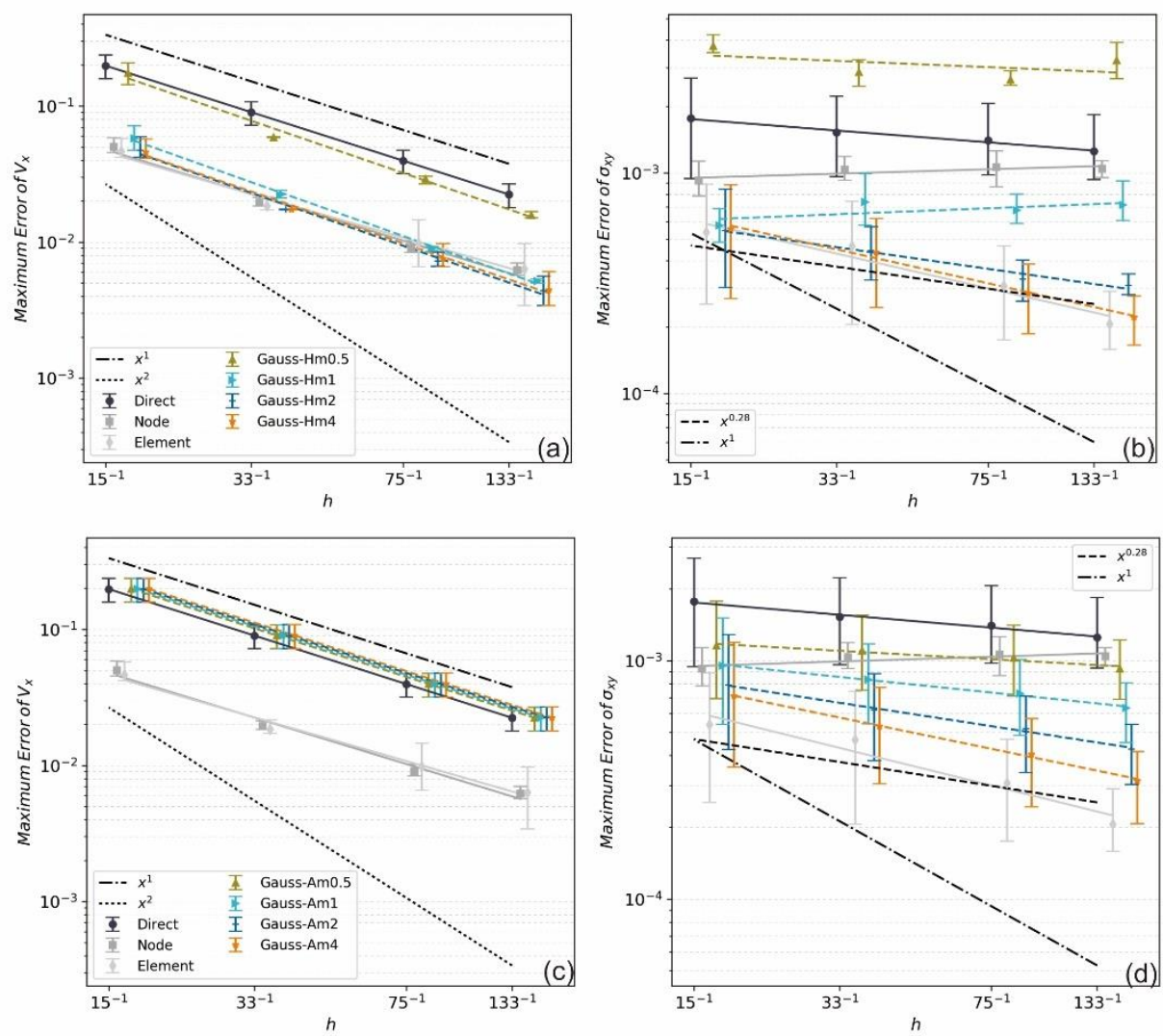

Figure A2. The $V_{x}(a \& c)$ and $\sigma_{x y}(b \& d)$ maximum error calculated with $\mathrm{Q}_{1}$ elements for different preprocessing methods applied to the simple shear model with distorted mesh. Every row shares the same legend. The black dashed line in the right panel $(b \& d)$ has the same slope of the Gaussian-quadrature-point based method with a sampling radius of two element size. 

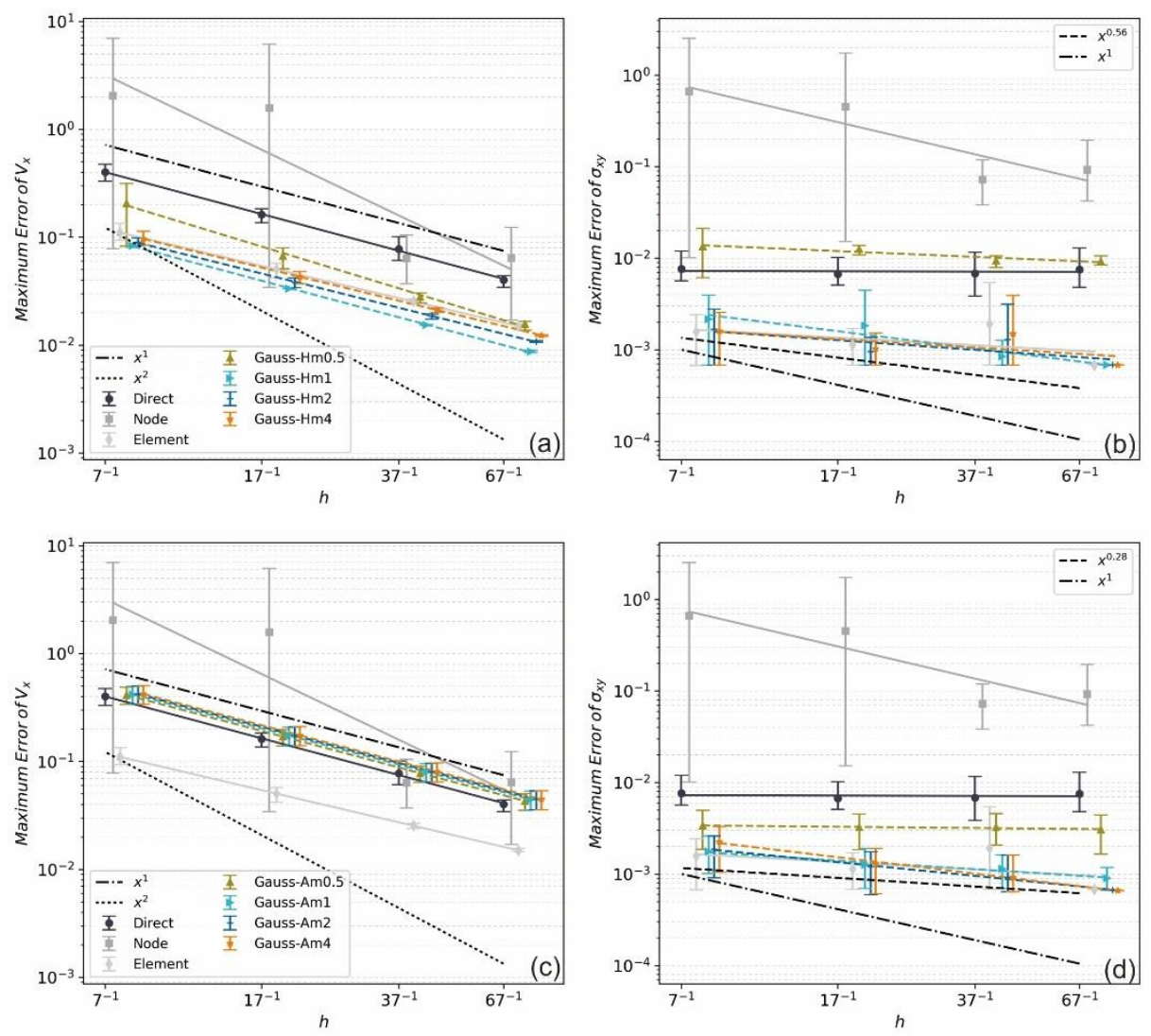

Figure A3. The $V_{x}(\mathrm{a} \& \mathrm{c})$ and $\sigma_{x y}(\mathrm{~b} \& \mathrm{~d})$ maximum error calculated with $\mathrm{Q}_{2}$ elements for different preprocessing methods applied to the simple shear model with distorted mesh. Every row shares the same legend. The black dashed line in the right panel $(b \& d)$ has the same slope of the Gaussian-quadrature-point based method with a sampling radius of one element size. 

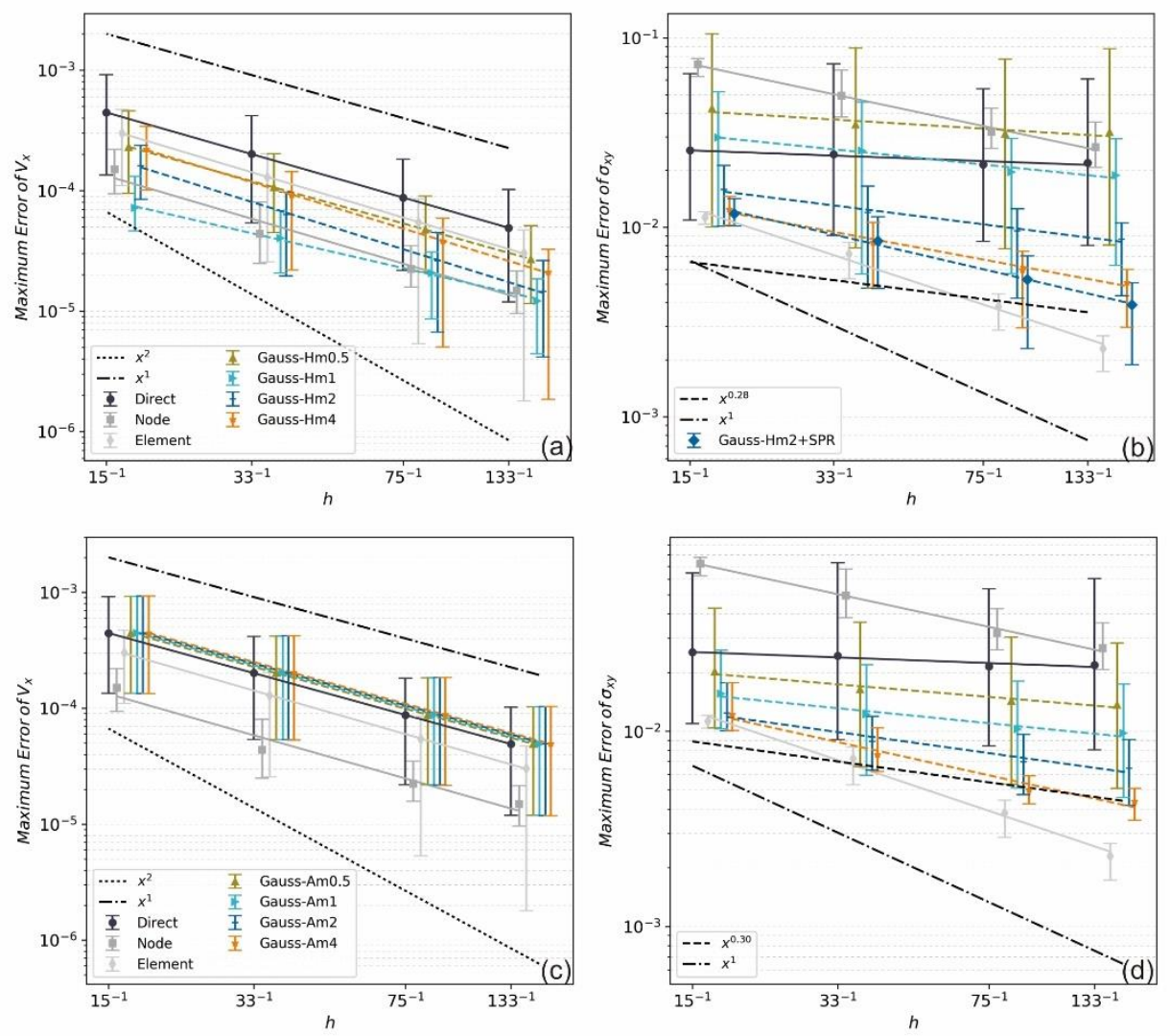

Figure A4. The $V_{x}(a \& c)$ and $\sigma_{x y}(b \& d)$ maximum error calculated with $\mathrm{Q}_{1}$ elements for different preprocessing methods applied to the SolCx benchmark model. Every row shares the same legend. The black dashed line in the right panel $(b \& d)$ has the same slope of the Gaussian-quadrature-point based method with a sampling radius of two element size. 

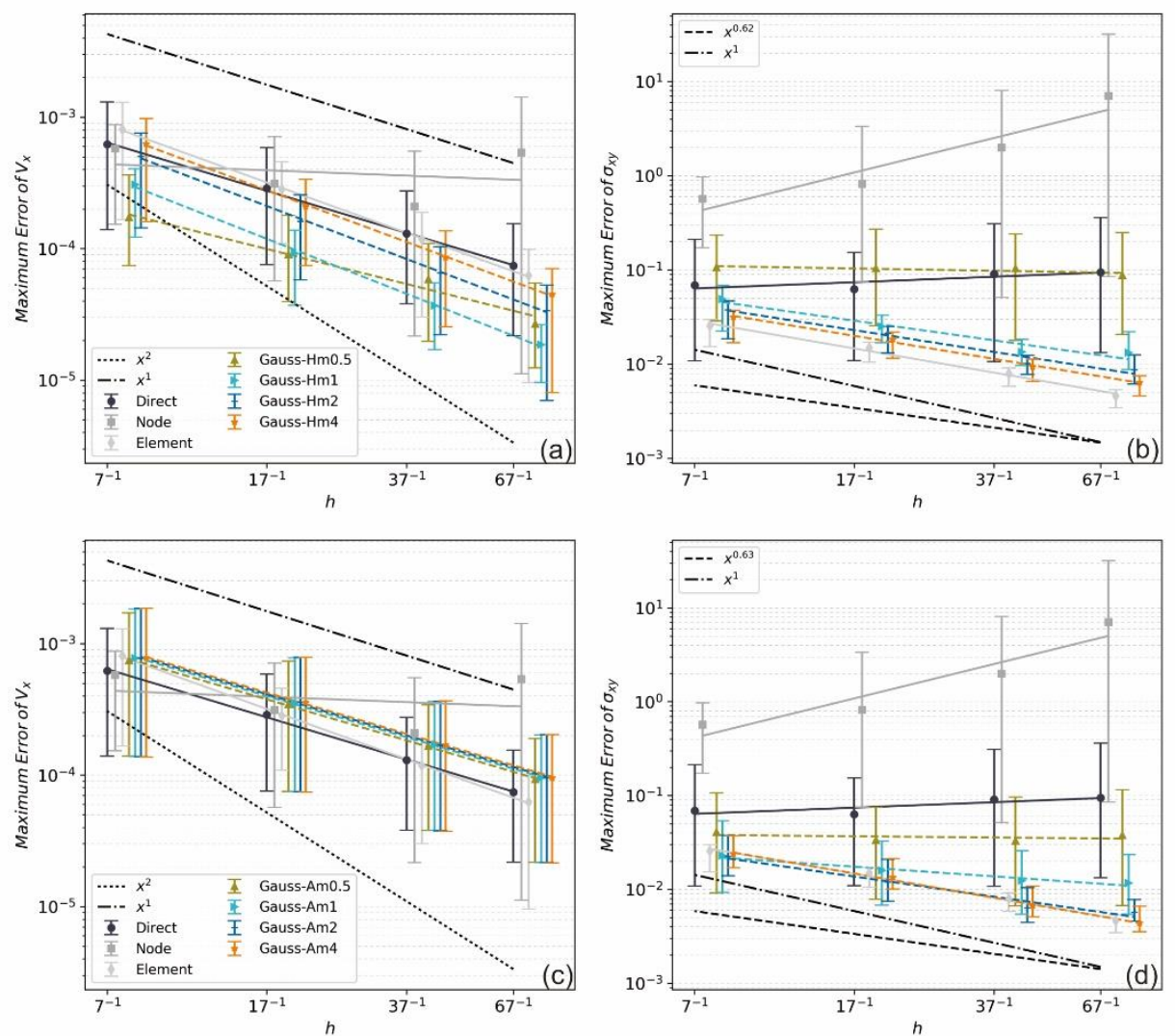

Figure A5. The $V_{x}(\mathrm{a} \& \mathrm{c})$ and $\sigma_{x y}(\mathrm{~b} \& \mathrm{~d})$ maximum error calculated with $\mathrm{Q}_{2}$ elements for different preprocessing methods applied to the SolCx benchmark model. Every row shares the same legend. The black dashed line in the right panel ( $b$ \& d) has the same slope of the Gaussianquadrature-point based method with a sampling radius of one element size. 
Table A1. Orders of accuracy of the RMS and Maximum errors between analytical and numerical solutions in velocity and shear stress for different smoothing methods.

\begin{tabular}{|c|c|c|c|c|c|c|c|c|c|}
\hline & & \multicolumn{4}{|c|}{$\overline{Q_{1}}$} & \multicolumn{4}{|c|}{$\overline{\mathrm{Q}_{2}}$} \\
\hline & & \multicolumn{2}{|c|}{ RMS } & \multicolumn{2}{|c|}{ Maximum } & \multicolumn{2}{|c|}{ RMS } & \multicolumn{2}{|c|}{ Maximum } \\
\hline & & $V_{x}$ & $\sigma_{x y}$ & $V_{x}$ & $\sigma_{x y}$ & $V_{x}$ & $\sigma_{x y}$ & $V_{x}$ & $\sigma_{x y}$ \\
\hline \multirow{3}{*}{ Simple } & Direct & 1 & 0.64 & 1 & -0.09 & 1.02 & 0.6 & 0.98 & -0.15 \\
\hline & Node & 1.02 & 0.53 & 1 & -0.04 & 1.08 & -0.15 & 0.36 & -1.09 \\
\hline & Element & 1.02 & 1.01 & 1 & 0.24 & 1.06 & 1.03 & 0.99 & 0.03 \\
\hline \multirow[t]{2}{*}{ shear } & Gauss-Hm0.5 & 1.01 & 0.54 & 1.02 & -0.04 & 1.04 & 0.52 & 1 & -0.18 \\
\hline & Gauss-Hm1 & 1.04 & 0.53 & 1.05 & -0.11 & 1.12 & 0.69 & 1.03 & -0.07 \\
\hline with & Gauss-Hm2 & 1.06 & 0.6 & 1.04 & -0.04 & 1.08 & 0.95 & 1 & 0.02 \\
\hline \multirow{2}{*}{ regular } & Gauss-Hm4 & 1.04 & 0.77 & 1.01 & 0.18 & 1.06 & 1 & 0.99 & 0.03 \\
\hline & Gauss-Am0.5 & 1 & 0.7 & 1 & 0.08 & 1.02 & 0.72 & 1.01 & 0.01 \\
\hline \multirow[t]{4}{*}{ mesh } & Gauss-Am1 & 1 & 0.77 & 1 & 0.13 & 1.03 & 0.96 & 1.02 & 0.08 \\
\hline & Gauss-Am2 & 1 & 0.87 & 1 & 0.21 & 1.03 & 1.06 & 1.02 & 0 \\
\hline & Gauss-Am4 & 1 & 0.94 & 1 & 0.26 & 1.03 & 1.07 & 1.02 & 0 \\
\hline & Direct & 1.21 & 0.67 & 1 & 0.15 & 1.32 & 0.75 & 1.03 & 0.01 \\
\hline \multirow{2}{*}{ Simple } & Node & 1.22 & 0.73 & 0.96 & -0.06 & 2.49 & 2.01 & 1.87 & 1.08 \\
\hline & Element & 1.44 & 0.72 & 0.9 & 0.44 & 1.52 & 0.56 & 0.91 & 0.24 \\
\hline \multirow[t]{2}{*}{ shear } & Gauss-Hm0.5 & 1.37 & 0.92 & 1.08 & 0.08 & 1.78 & 0.84 & 1.17 & 0.19 \\
\hline & Gauss-Hm1 & 1.37 & 0.72 & 1.11 & -0.08 & 1.99 & 0.94 & 1.03 & 0.58 \\
\hline with & Gauss-Hm2 & 1.46 & 0.72 & 1.09 & 0.28 & 1.74 & 0.74 & 0.96 & 0.31 \\
\hline \multirow{2}{*}{ distorted } & Gauss-Hm4 & 1.47 & 0.73 & 1.07 & 0.43 & 1.63 & 0.67 & 0.93 & 0.28 \\
\hline & Gauss-Am0.5 & 1.18 & 0.74 & 1 & 0.1 & 1.26 & 0.73 & 1.03 & 0.04 \\
\hline \multirow[t]{8}{*}{ mesh } & Gauss-Am1 & 1.2 & 0.8 & 1 & 0.18 & 1.26 & 0.93 & 1.02 & 0.29 \\
\hline & Gauss-Am2 & 1.21 & 0.85 & 1 & 0.28 & 1.28 & 0.95 & 1.02 & 0.45 \\
\hline & Gauss-Am4 & 1.21 & 0.87 & 1 & 0.37 & 1.28 & 0.97 & 1.02 & 0.54 \\
\hline & Direct & 1.01 & 0.71 & 1.01 & 0.08 & 0.95 & 0.65 & 0.98 & -0.18 \\
\hline & Node & 0.96 & 0.96 & 1.04 & 0.47 & 1 & -0.03 & 0.12 & -1.12 \\
\hline & Element & 1.09 & 0.84 & 1.05 & 0.73 & 1.31 & 0.87 & 1.16 & 0.78 \\
\hline & Gauss-Hm0.5 & 1.04 & 0.72 & 0.98 & 0.14 & 0.93 & 0.73 & 0.81 & 0.07 \\
\hline & Gauss-Hm1 & 0.91 & 0.77 & 0.81 & 0.23 & 1.4 & 0.99 & 1.27 & 0.63 \\
\hline \multirow[t]{6}{*}{ SolCx } & Gauss-Hm2 & 1.15 & 0.8 & 1.1 & 0.28 & 1.37 & 0.94 & 1.22 & 0.7 \\
\hline & Gauss-Hm4 & 1.13 & 0.82 & 1.08 & 0.41 & 1.35 & 0.9 & 1.2 & 0.74 \\
\hline & Gauss-Am0.5 & 1.01 & 0.76 & 1.01 & 0.18 & 0.9 & 0.72 & 0.95 & 0.04 \\
\hline & Gauss-Am1 & 1.01 & 0.82 & 1.01 & 0.22 & 0.9 & 0.89 & 0.95 & 0.31 \\
\hline & Gauss-Am2 & 1.01 & 0.88 & 1.01 & 0.3 & 0.9 & 0.91 & 0.95 & 0.65 \\
\hline & Gauss-Am4 & 1.01 & 0.92 & 1.01 & 0.48 & 0.9 & 0.9 & 0.95 & 0.79 \\
\hline
\end{tabular}

